\title{
Thermobarometry, Geochronology and the Interpretation of $P-T-t$ Data in the Britt Domain, Ontario Grenville Orogen, Canada
}

\author{
by M. E. TUCCILLO, K. MEZGER*, E. J. ESSENE, \\ AND B. A. VAN DER PLUIJM \\ Department of Geological Sciences, University of Michigan, Ann Arbor, Michigan 48109
}

(Received 2 April 1991; revised typescript accepted 3 February 1992)

\begin{abstract}
A BSTRACT
Textural evidence, thermobarometry, and geochronology were used to constrain the pressuretemperature-time $(P-T-t)$ history of the southern portion of the Britt domain in the Central Gneiss Belt, Ontario Grenville Province. Typical metapelitic assemblages are quartz + plagioclase + biotite + garnet + kyanite \pm alkali feldspar \pm sillimanite \pm rutile \pm ilmenite \pm staurolite \pm gahnite \pm muscovite. Metatonalitic assemblages have quartz + plagioclase + garnet + biotite + hornblende + rutile + ilmenite. Metagabbroic rocks contain plagioclase + garnet + clinopyroxene + biotite + ilmenite \pm hornblende \pm rutile \pm quartz. Notable textural features include overgrowths of sillimanite on kyanite and of spinel on staurolite. The spinel overgrowths can be modeled by the breakdown of staurolite via the reaction $\mathrm{Fe}$-staurolite $=$ hercynite + kyanite + quartz $+\mathrm{H}_{2} \mathrm{O}$. The decomposition of staurolite to hercynite has a steep $\mathrm{d} P / \mathrm{d} T$ slope and constrains the late prograde path of a staurolite metapelite. Garnet- $\mathrm{Al}_{2} \mathrm{SiO}_{5}$-plagioclase-quartz (GASP) barometry applied to metapelitic garnets that preserve calcium zoning reveals a pressure decrease from $\sim 11$ to $6 \mathrm{~kb}$ at an assumed temperature of $700^{\circ} \mathrm{C}$. Garnet-plagioclase-ilmenite-rutile-quartz and garnet-clinopyroxene-plagioclase-quartz barometry is in good agreement with pressures obtained with the GASP barometer. Geochronologic data from garnet, allanite, and monazite in metapelitic rocks give ages that fall into two groups, $\sim 1.4 \mathrm{Ga}$ and $\sim 1.1 \mathrm{Ga}$, suggesting the presence of at least two metamorphic events in the area. It is most reasonable to assign the $1.4 \mathrm{Ga}$ age to the high-pressure data and the $1.1 \mathrm{Ga}$ age to the lower-pressure data. Collectively the $P-T-t$ data indicate a complex and protracted history rather than a single cycle of burial and uplift for this part of the Grenville Province.
\end{abstract}

\section{INTRODUCTION}

One of the aims of metamorphic petrology is to gain information about the pressure and temperature histories of terranes through the use of mineral equilibria. Chemical analyses of minerals assumed to be in equilibrium have been used in conjunction with barometers and thermometers to estimate the pressure $(P)$ and temperature $(T)$ conditions attained at or near the peak of metamorphism (Ghent et al., 1982; Chamberlain \& Lyons, 1983; St.-Onge, 1984; Bohlen et al., 1985; Sandiford, 1985; Moecher et al., 1986; Essene, 1989; Anovitz \& Essene, 1990; Mezger et al., 1990). In addition, chemical zonation in minerals has been used to estimate the $P-T$ conditions at various stages in the metamorphic histories of rocks (Savage \& Sills, 1980; Brown \& Earle, 1983; Spear \& Selverstone, 1983; Hodges \& Royden, 1984; Selverstone et al., 1984; Spear et al., 1984; Selverstone \& Spear, 1985; St.-Onge, 1987; St.-Onge \& King, 1987; Harley, 1988; Perchuk et al., 1989). These $P-T$ paths are considered

* Present address: Max-Planck Institut für Chemie, Saarstraße, 23, Postfach 3060, D-6500, Mainz, FRG 
to be indicative of the tectonic history of a metamorphic terrane (e.g., England \& Thompson, 1984; Thompson \& England, 1984; Spear, 1989). At greenschist to middle amphibolite facies conditions, zonation is generally preserved in many minerals involved in reactions that can be used to obtain prograde and retrograde $P-T$ information. At higher grades of metamorphism, however, the amount and nature of information to be gained become more limited because of chemical re-equilibration of these minerals. For example, growth zoning in garnet may become eradicated by volume diffusion when temperatures exceed $\sim 650^{\circ} \mathrm{C}$ (e.g., Woodsworth, 1977; Yardley, 1977), and cation exchange reactions, often used as indicators of temperature, are particularly susceptible to resetting as rocks cool from peak temperatures (Bohlen \& Essene, 1980; Barnicoat, 1983; Indares \& Martignole, 1985a; Edwards \& Essene, 1988; Frost \& Chacko, 1989; Perkins, 1990).

Attempts to use thermobarometry to constrain $P-T$ paths of high-grade rocks often involve pairing core and rim analyses of coexisting minerals (e.g., Tracy, 1982; Barnicoat, 1983; Brown \& Earle, 1983; Harley, 1988; Anovitz \& Essene, 1990). The cores are assumed to represent peak or near-peak metamorphic conditions, whereas the rims have reset during early stages of retrogression. However, problems may arise because early retrograde temperatures of high-grade rock may still be high enough to permit homogenization and cation exchange of $\mathrm{Fe}$ and $\mathrm{Mg}$ between garnet and other ferromagnesian phases, causing exchange thermometers, and in some cases barometers, to be variably reset during retrogression (Frost \& Chacko, 1989; Selverstone \& Chamberlain, 1990). Temperatures recorded, therefore, may not correspond to the pressures obtained from barometry. When this occurs, connecting core and rim pressures and temperatures may yield spurious $P-T$ points. Nonetheless, Bohlen (1990) concluded that it is possible to obtain reasonable $P-T$ estimates in high-grade rocks with careful selection of samples and application of thermobarometers.

Quantitative thermobarometry applied to mineral zoning may yield an apparently smooth $P-T$ path based on the assumption that the pressures and temperatures obtained represent one metamorphic episode. A simple $P-T$ path may, however, be erroneous if the observed mineral chemistries and zoning patterns are the result of more than one metamorphic episode. Interpretations of the significance of $P-T$ data for the evolution of an area are therefore greatly enhanced by information on the timing of metamorphism. For this study we have collected U-Pb chronologic data for garnet, monazite, and allanite. A garnet and a monazite were selected from a sample with garnets that show pronounced major element zoning (Tuccillo et al., 1990). The combined geochronologic and $P-T$ data provide evidence that the southern Britt domain has experienced more than one metamorphic event. Therefore, the assumption of a simple $P-T$ path for this area based only on pressure and temperature estimates is likely to be incorrect.

\section{REGIONAL GEOLOGY}

The Grenville Province in Ontario is bounded on the northwest by the Archean Superior Province and Early Proterozoic Southern Province, and on the southeast by the Appalachian Orogen (Fig. 1a). Radiometric age determinations show that the Ontario Grenville was metamorphosed in the Late Proterozoic (Easton, 1986; van Breemen et al., 1986). The major subdivisions of the Grenville Orogen are, from northwest to southeast, the Grenville Front Tectonic Zone (GFTZ), the Central Gneiss Belt (CGB), the Central Metasedimentary Belt (CMB), and the Central Granulite Terrane (CGT) (Fig. 1a; Wynne-Edwards, 1972; Davidson, 1986). 

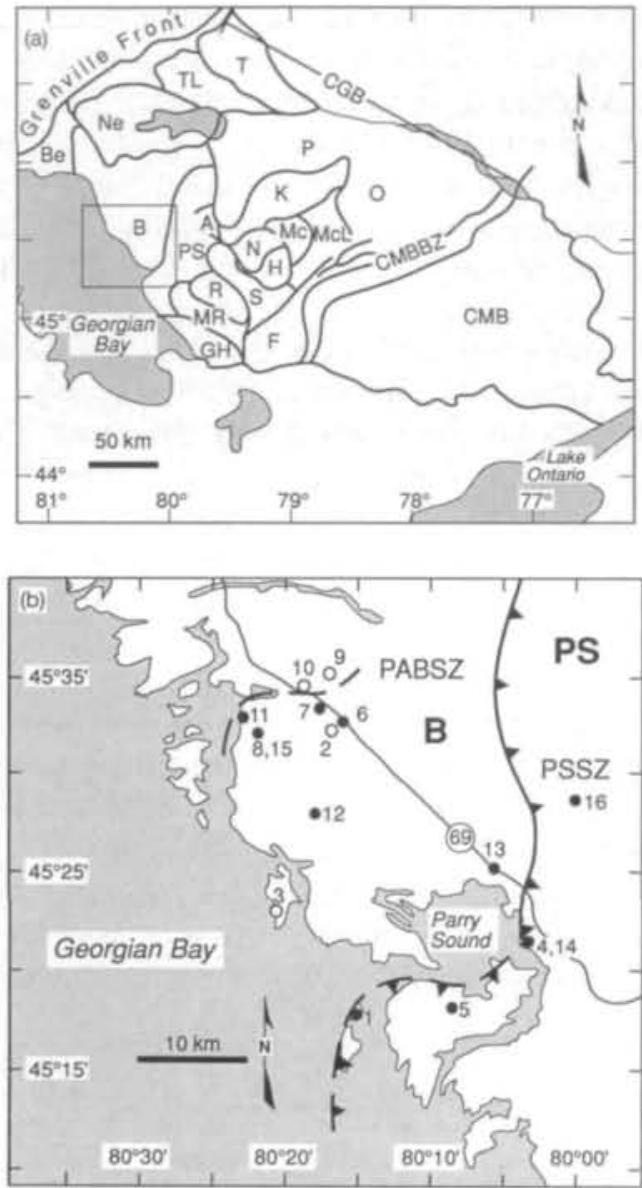

FIG. I(a) Lithotectonic map of the Grenville Orogen in Ontario (R. M. Easton, written comm., 1990). A-Ahmic domain, B-Britt domain, Be-Beaverstone Bay domain, CGB-Central Gneiss Belt, CMB-Central Metasedimentary Belt, CMBBZ - Central Metasedimentary Belt Boundary Zone, F-Fishog domain, GH-Go Home domain, H-Huntsville domain, Mc-McCraney domain, McL-McLintock domain, MR-Moon River domain, $\mathrm{N}$-Novar domain, NE-Nepawassi domain, O-Opeongo domain, P-Powassan domain, PS-Parry Sound domain, R-Rosseau domain, S-Seguin domain, T-Tomiko domain, TL-Tilden Lake domain. Box shows area of detall in (b). (b) Location map for samples from Britt domain and Parry Sound Shear Zone, modified from Davidson et al. (1982) and Culshaw et al. (1988). Sample localities are marked by circles: O-meta-igneous rock; - - metasedimentary rock. PS-Parry Sound domain, B-Britt domain, PSSZ-Parry Sound Shear Zone, PABSZ-Pointe-au-Baril Shear Zone.

The Central Gneiss Belt consists of highly deformed, upper amphibolite to granulite facies gneisses of both supracrustal and plutonic origin (Davidson, 1984), and is further subdivided into domains based on distinctive lithologies, structural styles, and geophysical characteristics (Davidson et al., 1982). Field mapping shows that the domains are separated by ductile shear zones with shear-sense indicators that give a predominantly northwest sense of transport. This led Davidson (1984) to propose a tectonic model of deep-level, northwestdirected thrusting for the Central Gneiss Belt. Schwerdtner (1987) suggested that the shear zones may be large décollement horizons associated with buckling due to lateral compression. Pressures of $9-11 \mathrm{~kb}(0-9-1 \cdot 1 \mathrm{GPa})$ and temperatures of $700-800^{\circ} \mathrm{C}$ were reported 
by Anovitz \& Essene (1990) for this part of the Ontario Grenville Orogen. This study concentrates on the Britt domain, which forms the northwest part of the CGB.

The Britt domain is considered to be the lowest exposed structural slice in the Central Gneiss Belt. It is bounded to the northwest by the Grenville Front Tectonic Zone and to the southeast by the Parry Sound domain. The Parry Sound Shear Zone, which separates the Britt and Parry Sound domains, is a major northwest-directed ductile shear zone (Davidson, 1984). Detailed mapping of the Britt domain along Georgian Bay was conducted by Culshaw et al. (1988).

The petrology and chronology of the southern Britt domain are examined in detail for this paper. The dominant rock types in this area are pink and gray granitic gneisses with rare metapelitic rocks. These units are interspersed with granitoid plutons and mafic dikes (Culshaw et al., 1988) and are migmatitic in many places. The northeast-striking Pointe-auBaril Shear Zone (PABSZ) shown in Fig. 1b was mapped by Culshaw et al. (1988) on islands in Georgian Bay. Characteristic rock types in the shear zone include a gray metapelitic unit with fibrous sillimanite and wine-red garnet as well as mylonitized gneisses. In the course of our field studies the zone was traced on land and further east across Highway 69 (Fig. 1b).

Thermobarometric data for the southeastern Britt domain are sparse. Anovitz \& Essene (1990) obtained pressure and temperature estimates for only a few samples in the domain; their $P-T$ data are similar to values they obtained in the adjacent Parry Sound domain. This study was undertaken to determine the metamorphic conditions experienced by the Britt domain relative to the Parry Sound domain and to gain a more complete undertstanding of the tectonic evolution of this part of the Grenville Orogen using pressure, temperature, and geochronologic data. Approximately 50 thin sections were examined initially, and a dozen samples with assemblages appropriate for thermobarometry were selected for more detailed study (Table 1).

\section{ANALYTICAL METHODS}

Chemical data were obtained using wavelength-dispersive analysis (WDA) with the Cameca Camebax electron microprobe analyzer at the University of Michigan. An accelerating voltage of $15 \mathrm{kV}$, a sample current of $10 \mathrm{nA}$, a mixture of synthetic and natural mineral standards, and PAP software provided by Cameca were used. Garnets, pyroxenes, ilmenites, staurolites, and spinels were analyzed with a point beam. The beam was rastered over a $9-\mu \mathrm{m}^{2}$ area for feldspars and biotites to minimize the loss of $\mathrm{Na}$. Only the elements $\mathrm{Si}$, $\mathrm{Al}, \mathrm{Fe}, \mathrm{Mg}, \mathrm{Ca}$, and $\mathrm{Mn}$ were analyzed when mapping garnets for compositional variations because complete garnet analyses showed negligible amounts of $\mathrm{Ti}$ and $\mathrm{Cr}$ (Table 2).

Allanite, garnet, and monazite were obtained from metapelitic gneisses and sphene was extracted from a calc-silicate gneiss (samples 13) and a quartz-sphene vein (sample 14). Garnet and monazite separates were purified with heavy liquid and a Frantz Isodynamic separator. About $100 \mathrm{mg}$ of inclusion-free garnet fragments and one to six monazite grains were hand-picked under a binocular microscope for each analysis. The allanite is a centimeter-sized crystal and only small fragments were used for analysis. The sphenes in sample 13 were $0.2-0.5 \mathrm{~cm}$ in diameter; those in sample 14 were $\sim 5 \mathrm{~cm}$ in diameter and were picked after crushing. The allanite fragments and the monazite grains were washed in warm purified water. The garnets and sphenes were washed in warm, twice distilled $2 \mathrm{~N} \mathrm{HCl}$ for $\sim 20 \mathrm{~min}$ to remove surface contamination. All minerals were spiked with a mixed ${ }^{205} \mathrm{~Pb} /{ }^{235} \mathrm{U}$ tracer before dissolution and were digested in 3-ml screw-top Teflon PFA ${ }^{\mathrm{R}}$ vials inside Krogh-style Teflon ${ }^{R}$ bombs at $220^{\circ} \mathrm{C}$. Leached $\mathrm{K}$-feldspar was analyzed for its $\mathrm{Pb}$ isotope composition from sample 8 , and leached muscovite was analyzed from sample 4 . The 


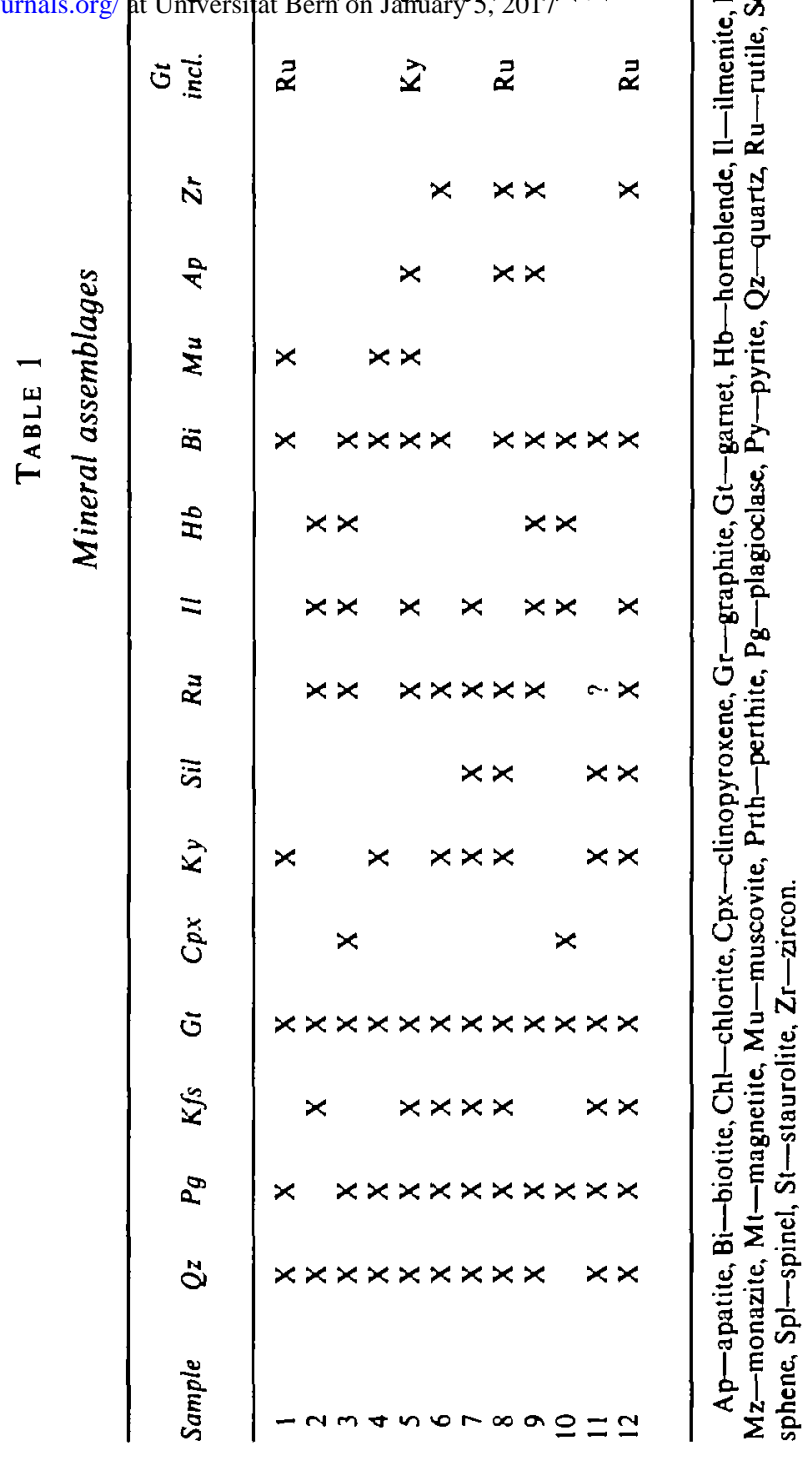




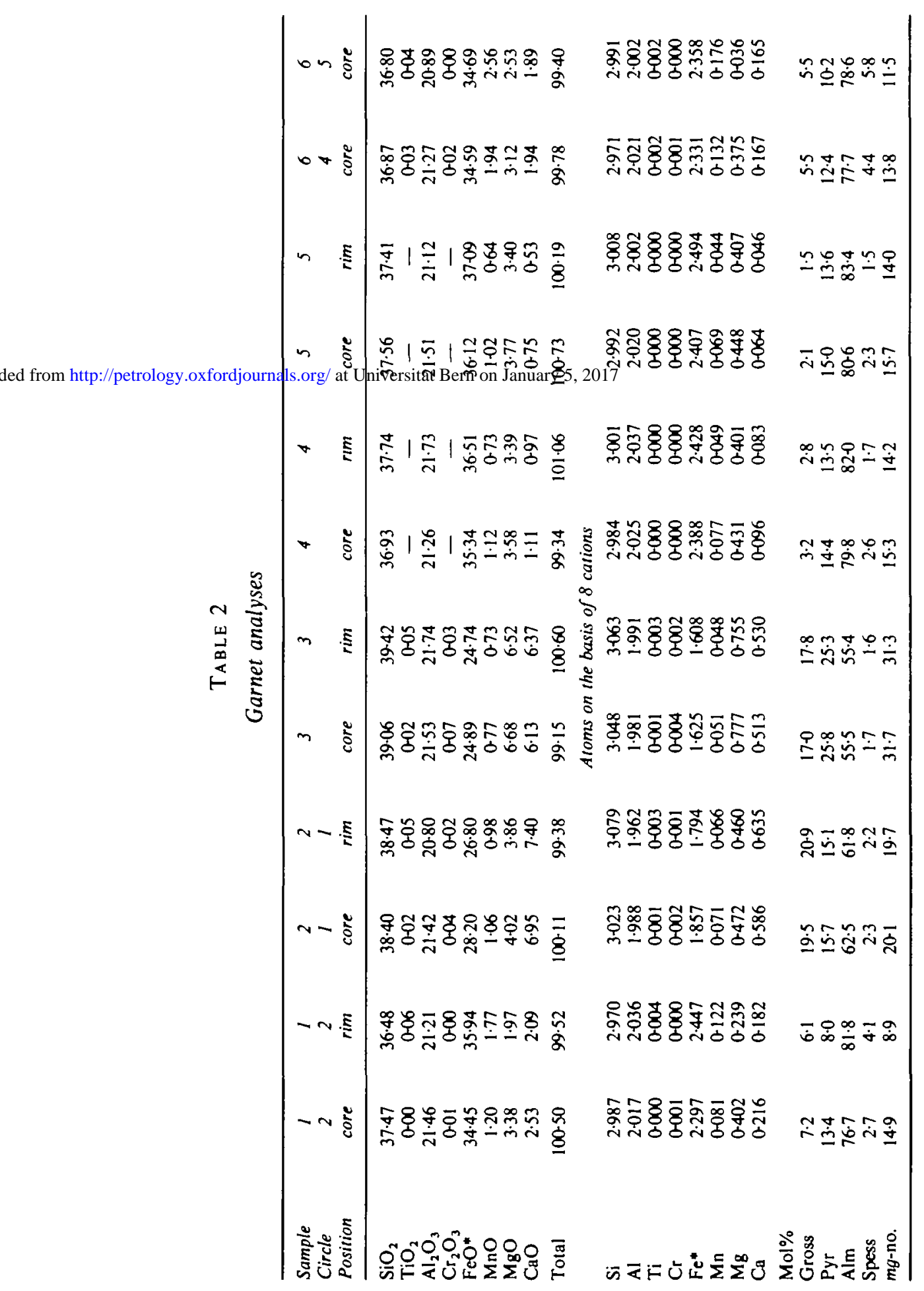




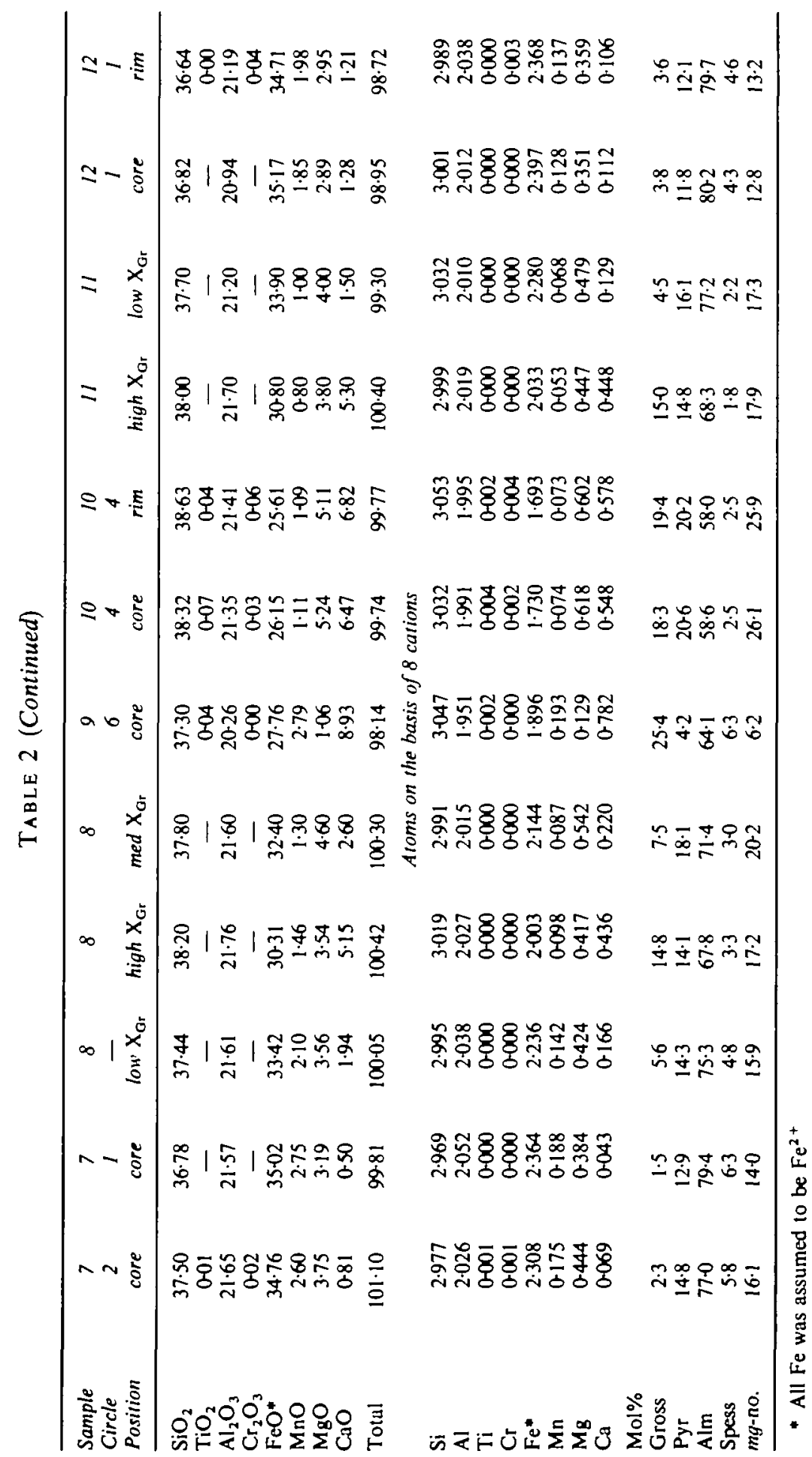


rock was crushed to $0.5-0.2 \mathrm{~mm}$, and the K-feldspar and muscovite were separated with heavy liquids. About $20 \mathrm{mg}$ of fresh, inclusion-free material was hand-picked under the binocular microscope. This was washed in boiling $6 \mathrm{~N} \mathrm{HNO}_{3}$ and in $6 \mathrm{~N} \mathrm{HCl}$ for at least 30 min and then leached with HF.

The total procedural blank was $40-150 \mathrm{pg}$ for $\mathrm{Pb}$ and $<20 \mathrm{pg}$ for U. Analyses were performed in the Radiogenic Isotope Laboratory at the University of Michigan using two VG Sector multicollector mass spectrometers equipped with a Daly knob or ion counting system. All $\mathrm{Pb}$ isotopic ratios were corrected with a mass discrimination factor of $0.13 \%$ per amu based on analyses of standards NBS-SRM 981 and NBS-SRM 983. The reproducibility of the ${ }^{207} \mathrm{~Pb} /{ }^{206} \mathrm{~Pb}$ ratio for the standard was $000 \%(2 \sigma)$. Based on repeated analyses of NBS-SRM U-500 the discrimination factor for $U$ was $0 \cdot 1-0.2 \%$ per amu depending on the batch of graphite solution used. [See Mezger et al. (1989) for further details on the analytical procedures.]

\section{PETROGRAPHY}

\section{Metapelites}

Metapelites from the Britt domain typically contain the primary mineral assemblage quartz + plagioclase + alkali feldspar + garnet + kyanite + sillimanite + biotite + rutile \pm ilmenite \pm zircon \pm staurolite \pm gahnite \pm monazite \pm apatite \pm sphalerite. If present, muscovite is intergrown with biotite, often crosscutting foliation and, in some cases, overgrowing kyanite; these relationships suggest that muscovite growth occurred during the retrograde history. Chlorite is a late alteration phase that replaces biotite. The metapelites are moderately to strongly foliated as defined by the preferred orientation of biotite. Although many biotite grains are clearly in the plane of foliation, others appear to have grown out of that plane and are interpreted to be retrograde. Some late biotite is observed overgrowing garnet and ragged kyanite (Fig. 2b). Biotite overgrowths on garnet suggest resorption of garnet by the reaction

$$
\begin{gathered}
\text { almandine }+\mathrm{K} \text {-feldspar }+\mathrm{H}_{2} \mathrm{O}=\text { annite + sillimanite + quartz } \\
\mathrm{Fe}_{3} \mathrm{Al}_{2} \mathrm{Si}_{3} \mathrm{O}_{12}+\mathrm{KAlSi}_{3} \mathrm{O}_{8}+\mathrm{H}_{2} \mathrm{O}=\mathrm{KFe}_{3} \mathrm{AlSi}_{3} \mathrm{O}_{10}(\mathrm{OH})_{2}+\mathrm{Al}_{2} \mathrm{SiO}_{5}+2 \mathrm{SiO}_{2}
\end{gathered}
$$

In four of five samples from the Britt domain, late sillimanite overgrows blades of kyanite (Fig. 2a, b). The coexistence of kyanite (and/or sillimanite) and alkali feldspar suggests that the reaction

$$
\begin{gathered}
\text { muscovite }+ \text { quartz }=\mathrm{K} \text {-feldspar }+ \text { kyanite }+\mathrm{H}_{2} \mathrm{O} \\
\mathrm{KAl}_{3} \mathrm{Si}_{3} \mathrm{O}_{10}(\mathrm{OH})_{2}+\mathrm{SiO}_{2}=\mathrm{KAlSi}_{3} \mathrm{O}_{8}+\mathrm{Al}_{2} \mathrm{SiO}_{5}+\mathrm{H}_{2} \mathrm{O}
\end{gathered}
$$

has occurred. Attempts to recapture the former alkali feldspar composition by reintegration yielded low $\left(500-600^{\circ} \mathrm{C}\right)$ temperatures, indicating that the albite component of the former alkali feldspar was lost by external granule formation (Bohlen \& Essene, 1977). Many feldspars show evidence of external granule formation by preservation of thin albite-rich rims. Garnets are irregularly shaped and may display embayed edges (Fig. 3a); inclusions are randomly distributed and consist of quartz, biotite, rutile, zircon, plagioclase, sphalerite, ilmenite, kyanite, and, in some samples, sillimanite and staurolite.

Figure $3 b$ shows a back-scattered electron micrograph of a large garnet from sample 7 that contains inclusions of kyanite, sillimanite, and staurolite. The grain is irregular, with rounded protuberances and smaller satellite garnets. Clusters of inclusions are observed in the rounded regions of the grain, suggestive of coalescence of smaller garnets into larger 

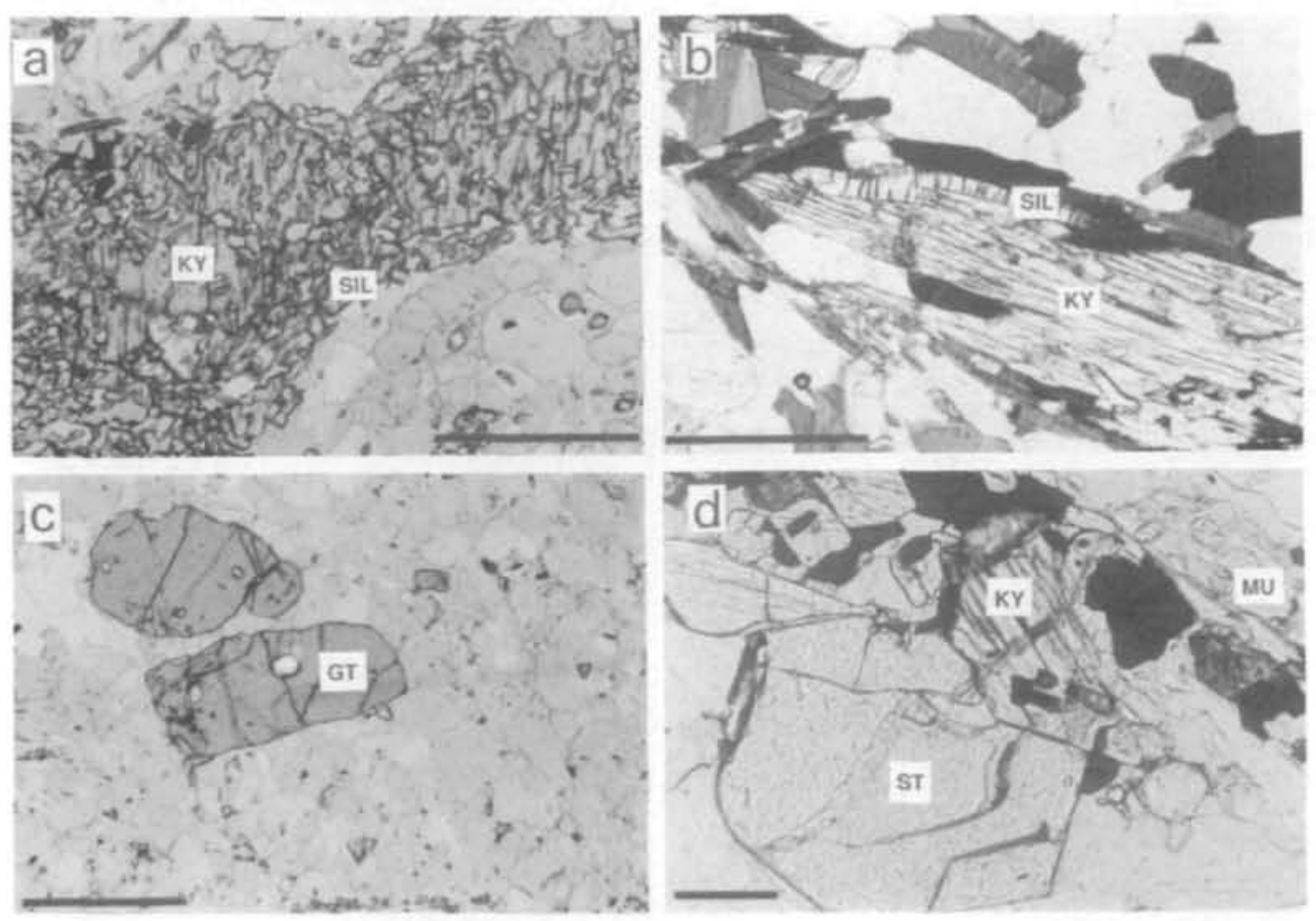

FiG. 2. Photomicrographs. (a,b) Textures of sillimanite overgrowing kyanite. Scale bars represent $0.5 \mathrm{~mm}$. (c) Tabular garnet, possibly a pseudomorph after staurolite. Matrix is predominantly feldspar. Scale bar represents $1 \mathrm{~mm}$. (d) Staurolite touching kyanite. Scale bar represents $0-1 \mathrm{~mm}$. Opaque mineral is ilmenite.

grains. Merging of smaller garnets to form larger grains is consistent with zoning patterns described by Tuccillo et al. (1990). Other garnets in the same sample are elongate and tabular and may be pseudomorphs after staurolite (Fig. 2c). This sample also shows striking textures of spinel surrounding staurolite, which indicate the prograde breakdown of staurolite to form spinel (Fig. 3c). Staurolite is seen touching garnet and kyanite (Fig. 2d), and spinel occurs in contact with the edge of one garnet (Fig. 3d). These relationships appear to record heterogeneous reactions, such as the growth of garnet and spinel at the expense of staurolite.

Three mica-rich pelitic schists from the Parry Sound Shear Zone (samples 1, 4, and 5) contain coarse muscovite intergrown with biotite. Garnets in these samples range in size from $0-1$ to $1 \mathrm{~cm}$ and have numerous inclusions (Fig. 3e), which are predominantly quartz, but also include biotite, rutile, and, in one sample (5), kyanite. Inclusions are most common in the centers of the garnet grains, leaving essentially inclusion-free rims. In samples 4 and 5 , biotite appears to be a primary phase; garnet overgrows biotite in sample 5 . In both samples coarse, abundant muscovite is intergrown with biotite and appears to be primary. The occurrence of texturally primary muscovite in these samples from the Parry Sound Shear Zone may indicate that this unit equilibrated under lower-grade conditions than the Britt domain. Muscovite in sample 4 overgrows blades of deformed kyanite. In sample 1 the garnets are flanked by dense mats of biotite, but the timing of biotite growth is not clear, as blades are observed overgrowing garnet and kyanite and vice versa. 

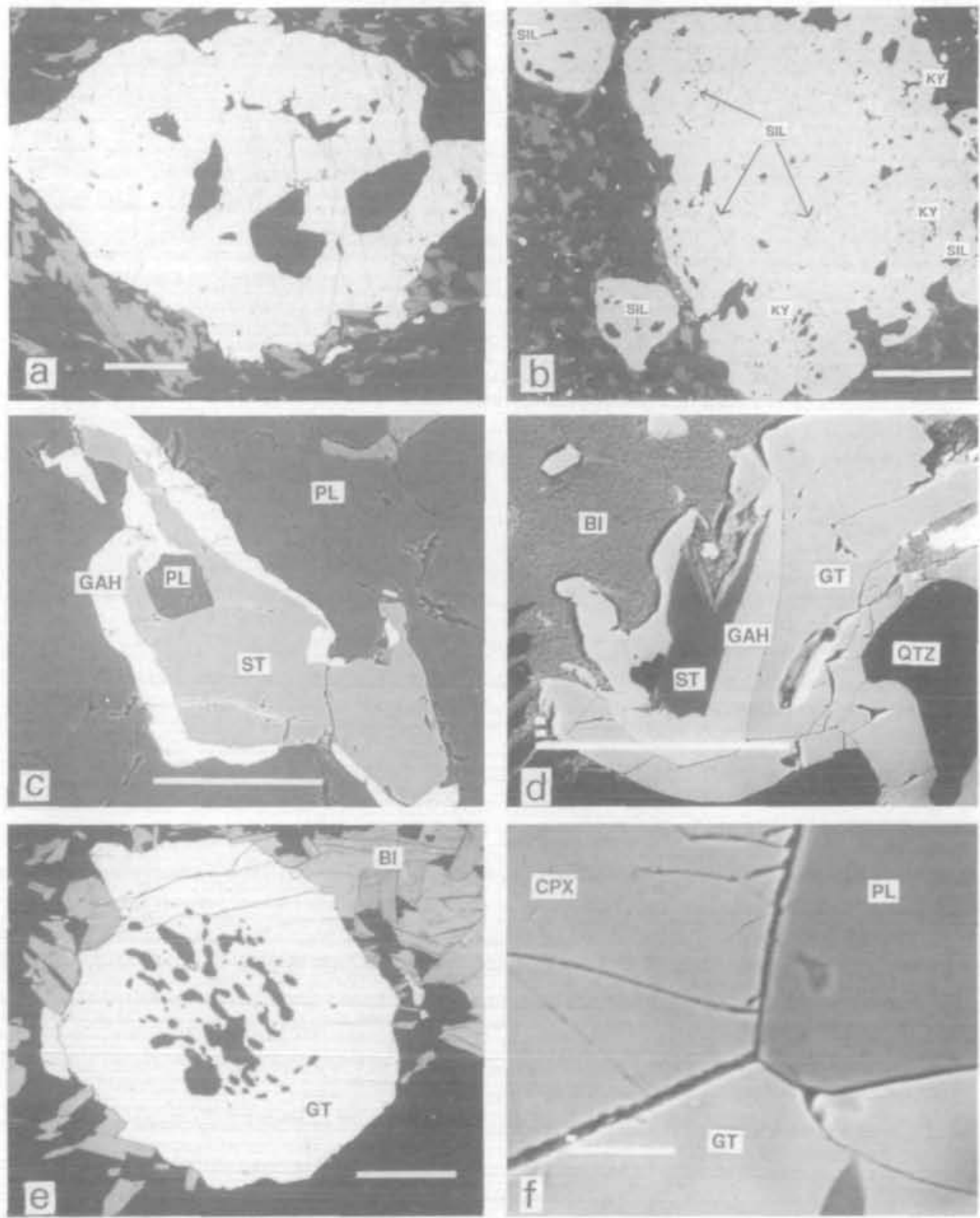

FIG. 3. Back-scattered electron micrographs. (a) Metapelitic garnet from Britt domain, sample 8. Scale bar represents $1 \mathrm{~mm}$. (b) Large garnet from sample 7 with inclusions of kyanite, sillimanite, staurolite, and quartz. Scale bar represents $1.5 \mu \mathrm{m}$. (c) Spinel rimming staurolite. Scale bar represents $100 \mu \mathrm{m}$. (d) Spinel surrounding staurolite and touching garnet. Bright minerals are sphalerite and pyrite. Scale bar represents $100 \mu \mathrm{m}$. (e) Garnet from Parry Sound Shear Zone (sample 4) showing cluster of inclusions in center of grain. Scale bar represents $0-5 \mathrm{~mm}$. (f) Triple junction of garnet, clinopyroxene, and plagioclase from sample 3 . Scale bar represents $10 \mu \mathrm{m}$. 


\section{Metatonalite}

One garnet-bearing metatonalite sample contains the GRIPS barometric assemblage and was selected for further study. Sample 9 is a well-foliated tonalitic gneiss with the assemblage quartz + plagioclase + garnet + biotite + hornblende + rutile + ilmenite + apatite + zircon. In this sample, biotite, which overgrows hornblende, defines the foliation. Irregular, ragged garnets contain inclusions of ilmenite, rutile, hornblende, and quartz.

\section{Metagabbros}

Three garnet-bearing metagabbroic rocks (samples 2,3 , and 10) were included in this study. The typical assemblage is plagioclase + garnet + clinopyroxene + ilmenite + biotite + hornblende \pm magnetite \pm rutile \pm quartz. These rocks are fine grained (average grain size $<0.5 \mathrm{~mm}$ ) and are unfoliated with abundant ragged hornblende, which is generally overgrown by biotite. Garnets are small and commonly occur in clusters. They are rounded or irregularly shaped with embayed edges and may have inclusions of plagioclase, magnetite, and biotite. Clinopyroxene also occurs in small rounded grains. Sample 2 also has larger grains of clinopyroxene with 'exsolved' ilmenite. These grains may be rimmed by garnet and plagioclase, ragged hornblende, or magnetite. Coarse, irregular magnetite is commonly surrounded by garnet and hornblende.

\section{MINERAL CHEMISTRY}

\section{Garnets}

The application of thermobarometry to chemically zoned minerals is one of the most important means by which $P-T$ paths are derived. Because garnet is involved in many barometric and thermometric reactions, zoned garnets may provide a wealth of $P-T$ information. Therefore, all the garnets used in this study were examined by microprobe for evidence of growth and/or retrograde zoning.

Metapelitic and metatonalitic garnets are usually almandine-pyrope solid solutions with low grossular and spessartine contents (Table 2); those in sample 9 are almandine-grossular solid solutions. Garnets in samples 6,9, and 12 are unzoned; garnets in sample 7 show an increase in pyrope and a decrease in almandine where the grains are in contact with staurolite or spinel. The large garnet described in sample 7 (Fig. 3b) does not preserve any compositional variations between the areas with kyanite inclusions and the areas with sillimanite inclusions; this may represent subsequent resetting of the major element chemistry in the garnet during metamorphism. There is a slight decrease in grossular content at the edges of this garnet.

Garnets in metapelitic samples 8 and 11 display unusual patterns of zoning (Tuccillo et al., 1990). The garnets in these samples contain discrete regions of high grossular content which are crosscut by variations in the $\mathrm{Mg} /(\mathrm{Mg}+\mathrm{Fe})$ ratio (mg-number) and $\mathrm{Mn}$ parallel to the grain boundaries and around touching biotite grains (Fig. 4). The Ca zoning is interpreted to represent growth zoning, whereas the core to rim decrease in $m g$-number and increase in $\mathrm{Mn}$ are interpreted to be a retrograde diffusional effect (Tuccillo et al., 1990).

Preliminary analytical mapping was carried out for garnets in metapelite samples 1,4 , and 5. The compositional variations of the garnets mapped in samples 1,5 , and 11 are illustrated in Figs. 4 and 5. Sample 1 (Fig. 5) exhibits a zoning pattern that is common in high-grade garnets (e.g., Tracy et al., 1976; Tracy, 1982, and references therein); $M g$ decreases from core to rim and $\mathrm{Mn}$ and $\mathrm{Fe}$ increase. This pattern has been interpreted to be the result of homogenization accompanied by cation exchange with another ferromagnesian mineral, 

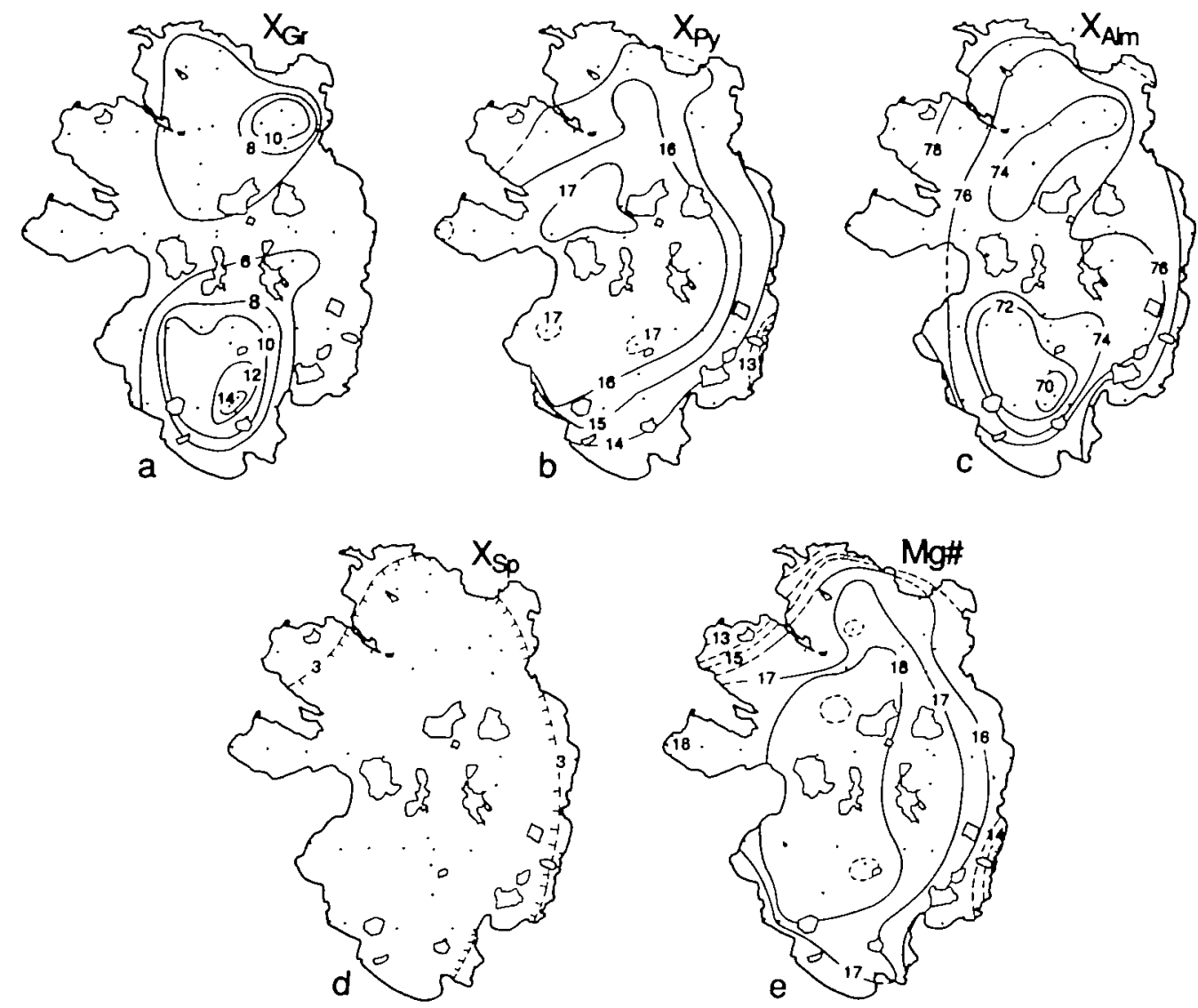

FIG. 4. Composition maps for garnet from sample 11. Each dot represents a microprobe analysis. X signifies mole percent. Garnet is $\sim 2.2 \mathrm{~mm} \times 2.9 \mathrm{~mm}$.

typically biotite, during retrogression (Grant \& Weiblen, 1971; Tracy et al., 1976; Anderson \& Olimpio, 1977; Woodsworth, 1977; Edwards \& Essene, 1988). The garnet mapped in sample 5 shows a decrease in $\mathrm{Mg}$ and increase in $\mathrm{Fe}$ from core to rim, but the spessartine content is slightly higher in the core than at the rim. The presence of higher $\mathrm{Mn}$ in the core may represent the remnants of growth zoning, whereas the other elements have completely reset (Hollister, 1966; Blackburn, 1969).

\section{Biotites}

Biotites in high-grade rocks have been shown to exchange $\mathrm{Mg}$ and $\mathrm{Fe}$ with garnets during cooling, which affects garnet-biotite thermometry (e.g., Indares \& Martignole, 1985a; Schreurs, 1985; Edwards \& Essene, 1988). The $\mathrm{Mg} / \mathrm{Fe}$ exchange with garnet may cause biotites touching garnets to have compositions different from those of matrix biotites; matrix biotites near garnets may have compositions different from those of distant biotites. Biotites were analyzed with particular care for textural relationships, between biotite and garnet (Table 5).

In sample 6, biotites show a decrease in $\mathrm{Mg} / \mathrm{Fe}$ (from 0.78 to 0-66) and an increase in $\mathrm{TiO}_{2}$ (from 2.8 to $3.9 \mathrm{wt} . \%$ ) away from garnet. Biotites in sample 1 also show a slight decrease in 

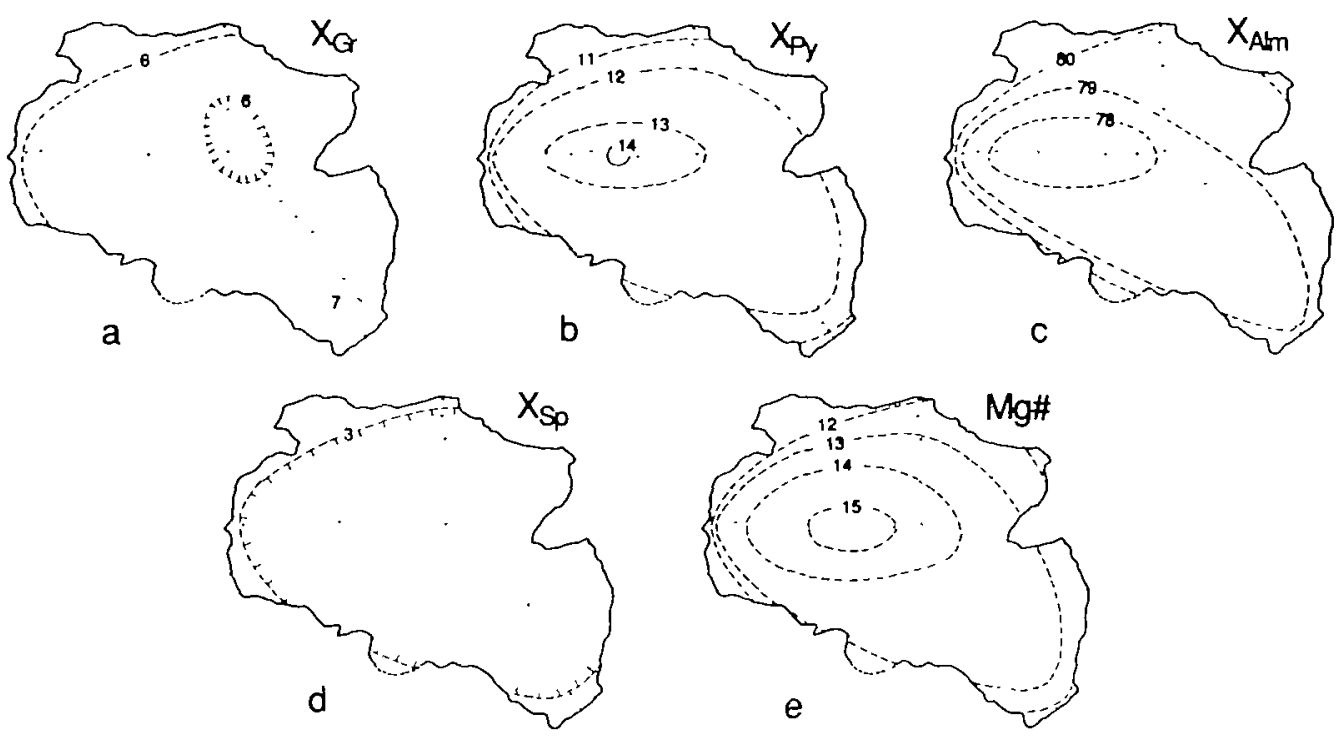

FIG. 5. Composition maps for garnet from sample 1 . Garnet is $\sim 1.2 \mathrm{~mm} \times 1.9 \mathrm{~mm}$. As only a small number of points were analyzed in this garnet the dashed contours have to be considered tentative.

$\mathrm{Mg} / \mathrm{Fe}$ (from 0.74 to 0.71 ) far away from the garnet; no variation was observed in $\mathrm{TiO}_{2}$ content. Analyses of biotites in samples 8 and 11 show no compositional variation with increasing distance from garnets. However, biotites that are included in or are touching garnets have substantially higher $\mathrm{Mg} / \mathrm{Fe}$ ratios and higher $\mathrm{Ti}$ than those not in contact with the garnets. In sample 8 , the average $\mathrm{Mg} / \mathrm{Fe}$ mole ratio for biotites inside garnets is $1 \cdot 30$. The

TABLE 3

Feldspar analyses

\begin{tabular}{|c|c|c|c|c|c|c|c|c|c|c|c|c|}
\hline $\begin{array}{l}\text { Sample } \\
\text { Circle } \\
\text { Position }\end{array}$ & $\begin{array}{c}1 \\
I \\
\text { core }\end{array}$ & $\begin{array}{c}1 \\
2 \\
\text { core }\end{array}$ & $\begin{array}{c}1 \\
2 \\
\text { rim }\end{array}$ & $\begin{array}{c}2 \\
\text { core }\end{array}$ & $\begin{array}{c}2 \\
\text { rim }\end{array}$ & $\begin{array}{c}3 \\
\text { core }\end{array}$ & $\begin{array}{c}3 \\
\text { rim }\end{array}$ & $\begin{array}{c}4 \\
\text { core }\end{array}$ & $\begin{array}{c}4 \\
\text { rim }\end{array}$ & $\begin{array}{c}5 \\
\text { core }\end{array}$ & $\begin{array}{c}5 \\
\mathrm{rim}\end{array}$ & $\begin{array}{c}6 \\
4 \\
\text { core }\end{array}$ \\
\hline $\begin{array}{l}\mathrm{SiO}_{2} \\
\mathrm{Al}_{2} \mathrm{O}_{3} \\
\mathrm{Fe}_{2} \mathrm{O}_{3} \\
\mathrm{CaO} \\
\mathrm{Na}_{2} \mathrm{O} \\
\mathrm{K}_{2} \mathrm{O}\end{array}$ & $\begin{array}{r}58.94 \\
25.03 \\
0-02 \\
6.45 \\
7.90 \\
0.22\end{array}$ & $\begin{array}{r}59 \cdot 50 \\
24.85 \\
0-04 \\
6 \cdot 25 \\
8 \cdot 11 \\
0-28\end{array}$ & $\begin{array}{r}59.01 \\
25.25 \\
0-07 \\
6.70 \\
7.97 \\
0.12\end{array}$ & $\begin{array}{r}60-49 \\
24 \cdot 37 \\
0-22 \\
6-01 \\
8.04 \\
0.31\end{array}$ & $\begin{array}{r}59 \cdot 85 \\
24.63 \\
0-42 \\
6.33 \\
7 \cdot 76 \\
0-31\end{array}$ & $\begin{array}{r}58 \cdot 12 \\
26 \cdot 17 \\
0.30 \\
7.94 \\
7 \cdot 16 \\
0.29\end{array}$ & $\begin{array}{r}56.88 \\
26.80 \\
0-40 \\
8.72 \\
6.51 \\
0.25\end{array}$ & $\begin{array}{r}60-84 \\
23 \cdot 42 \\
0-00 \\
5 \cdot 29 \\
8 \cdot 73 \\
0-07\end{array}$ & $\begin{array}{r}61 \cdot 12 \\
23 \cdot 63 \\
0-03 \\
5 \cdot 34 \\
8 \cdot 64 \\
0-03\end{array}$ & $\begin{array}{r}64.43 \\
22.58 \\
0-00 \\
3 \cdot 24 \\
9.84 \\
0-08\end{array}$ & $\begin{array}{r}64.91 \\
21.91 \\
0-08 \\
2.73 \\
9.87 \\
0.39\end{array}$ & $\begin{array}{r}61.48 \\
23.57 \\
0.14 \\
4.89 \\
8.67 \\
0.25\end{array}$ \\
\hline Total & \multicolumn{11}{|c|}{ Aroms on the basis of 8 oxygens } & $99-00$ \\
\hline $\begin{array}{l}\mathrm{Si} \\
\mathrm{Al} \\
\mathrm{Fe} \\
\mathrm{Ca} \\
\mathrm{Na} \\
\mathrm{K}\end{array}$ & $\begin{array}{l}2.666 \\
1.334 \\
0-001 \\
0-313 \\
0-693 \\
0-013\end{array}$ & $\begin{array}{l}2.678 \\
1.318 \\
0-001 \\
0-301 \\
0-708 \\
0-016\end{array}$ & $\begin{array}{l}2.656 \\
1 \cdot 340 \\
0-002 \\
0-323 \\
0-696 \\
0-007\end{array}$ & $\begin{array}{l}2 \cdot 707 \\
1 \cdot 286 \\
0-007 \\
0-288 \\
0-698 \\
0-018\end{array}$ & $\begin{array}{l}2 \cdot 687 \\
1 \cdot 303 \\
0-014 \\
0-304 \\
0-676 \\
0-018\end{array}$ & $\begin{array}{l}2.605 \\
1 \cdot 383 \\
0-010 \\
0-381 \\
0-622 \\
0-017\end{array}$ & $\begin{array}{l}2.565 \\
1.424 \\
0-014 \\
0.421 \\
0.569 \\
0-014\end{array}$ & $\begin{array}{l}2 \cdot 746 \\
1 \cdot 246 \\
0-000 \\
0-256 \\
0-764 \\
0-004\end{array}$ & $\begin{array}{l}2.744 \\
1.251 \\
0-001 \\
0.257 \\
0.752 \\
0-002\end{array}$ & $\begin{array}{l}2.835 \\
1 \cdot 171 \\
0-000 \\
0-153 \\
0.839 \\
0-004\end{array}$ & $\begin{array}{l}2.863 \\
1 \cdot 139 \\
0-003 \\
0-129 \\
0-844 \\
0-022\end{array}$ & $\begin{array}{l}2.754 \\
1.244 \\
0-005 \\
0-235 \\
0-753 \\
0-014\end{array}$ \\
\hline $\begin{array}{l}\text { Mol\% } \\
\text { Ab } \\
\text { An } \\
\text { Or }\end{array}$ & $\begin{array}{r}68-0 \\
30-7 \\
1 \cdot 3\end{array}$ & $\begin{array}{r}69 \cdot 1 \\
29 \cdot 3 \\
1.6\end{array}$ & $\begin{array}{r}67.8 \\
31.5 \\
0.7\end{array}$ & $\begin{array}{r}69.5 \\
28.7 \\
1.8\end{array}$ & $\begin{array}{r}67.7 \\
30-5 \\
1.8\end{array}$ & $\begin{array}{r}61.0 \\
37 \cdot 3 \\
1.7\end{array}$ & $\begin{array}{r}56.7 \\
41.9 \\
1.4\end{array}$ & $\begin{array}{r}74 \cdot 6 \\
25-0 \\
0-4\end{array}$ & $\begin{array}{r}74 \cdot 4 \\
25 \cdot 4 \\
0.2\end{array}$ & $\begin{array}{r}84 \cdot 2 \\
15 \cdot 3 \\
0 \cdot 4\end{array}$ & $\begin{array}{r}84 \cdot 8 \\
13 \cdot 0 \\
2 \cdot 2\end{array}$ & $\begin{array}{r}75 \cdot 1 \\
23 \cdot 4 \\
1.4\end{array}$ \\
\hline
\end{tabular}


TABle 3 (Continued)

\begin{tabular}{|c|c|c|c|c|c|c|c|c|c|c|c|}
\hline $\begin{array}{l}\text { Sample } \\
\text { Circle } \\
\text { Position }\end{array}$ & $\begin{array}{c}7 \\
1 \\
\text { core }\end{array}$ & $\begin{array}{c}7 \\
5 \\
\text { core }\end{array}$ & $\begin{array}{c}8 \\
\text { matrix }\end{array}$ & $\begin{array}{c}8 \\
\text { incl }\end{array}$ & $\begin{array}{c}9 \\
6 \\
\text { core }\end{array}$ & $\begin{array}{c}9 \\
6 \\
\text { rim }\end{array}$ & $\begin{array}{l}10 \\
\text { core }\end{array}$ & $\begin{array}{l}10 \\
\text { rim }\end{array}$ & $\begin{array}{c}I I \\
I \\
\text { core }\end{array}$ & $\begin{array}{c}12 \\
I \\
\text { core }\end{array}$ & $\begin{array}{c}12 \\
1 \\
\text { rim }\end{array}$ \\
\hline $\begin{array}{l}\mathrm{SiO}_{2} \\
\mathrm{Al}_{2} \mathrm{O}_{3} \\
\mathrm{Fe}_{2} \mathrm{O}_{3} \\
\mathrm{CaO} \\
\mathrm{Na}_{2} \mathrm{O} \\
\mathrm{K}_{2} \mathrm{O}\end{array}$ & $\begin{array}{r}66.15 \\
20-91 \\
0-06 \\
1.61 \\
10-81 \\
0-13\end{array}$ & $\begin{array}{r}66 \cdot 15 \\
20-89 \\
0-07 \\
1.57 \\
10-70 \\
0-15\end{array}$ & $\begin{array}{r}59 \cdot 39 \\
25-07 \\
0-03 \\
7 \cdot 12 \\
7 \cdot 58 \\
0-28\end{array}$ & $\begin{array}{r}58.79 \\
25.83 \\
0.13 \\
7.08 \\
7.36 \\
0.49\end{array}$ & $\begin{array}{r}63 \cdot 20 \\
22 \cdot 21 \\
0-06 \\
4-02 \\
9 \cdot 39 \\
0-39\end{array}$ & $\begin{array}{r}62 \cdot 26 \\
22 \cdot 42 \\
0.21 \\
4 \cdot 33 \\
9 \cdot 22 \\
0.28\end{array}$ & $\begin{array}{r}60-06 \\
24.68 \\
0-09 \\
6.26 \\
8.05 \\
0.25\end{array}$ & $\begin{array}{r}57.74 \\
26.13 \\
0.35 \\
8.21 \\
6.98 \\
0.19\end{array}$ & $\begin{array}{r}59 \cdot 14 \\
25 \cdot 06 \\
0-04 \\
6.58 \\
8-06 \\
0-28\end{array}$ & $\begin{array}{r}61 \cdot 84 \\
23.20 \\
0-18 \\
4.75 \\
8.80 \\
0-31\end{array}$ & $\begin{array}{r}60-47 \\
23.47 \\
0.57 \\
5 \cdot 14 \\
8.63 \\
0.34\end{array}$ \\
\hline Total & 99.67 & $99 \cdot 53$ & $99 \cdot 47$ & 99.68 & $99 \cdot 27$ & 98.72 & $99 \cdot 39$ & $99 \cdot 60$ & $99 \cdot 16$ & $99-08$ & 98.62 \\
\hline \multicolumn{12}{|c|}{ Atoms on the basis of 8 oxygens } \\
\hline $\begin{array}{l}\mathrm{Si} \\
\mathrm{Al} \\
\mathrm{Fe} \\
\mathrm{Ca} \\
\mathrm{Na} \\
\mathrm{K}\end{array}$ & $\begin{array}{l}2.914 \\
1.086 \\
0-002 \\
0-076 \\
0-923 \\
0-007\end{array}$ & $\begin{array}{l}2.914 \\
1-086 \\
0-002 \\
0-074 \\
0-915 \\
0-008\end{array}$ & $\begin{array}{l}2 \cdot 666 \\
1 \cdot 327 \\
0-000 \\
0-341 \\
0-661 \\
0-011\end{array}$ & $\begin{array}{l}2.636 \\
1.363 \\
0-003 \\
0-341 \\
0-643 \\
0-029\end{array}$ & $\begin{array}{c}2 \cdot 818 \\
1 \cdot 167 \\
0-002 \\
0-192 \\
0-812 \\
0-022\end{array}$ & $\begin{array}{l}2 \cdot 796 \\
1 \cdot 187 \\
0-007 \\
0-208 \\
0-803 \\
0-016\end{array}$ & $\begin{array}{l}2 \cdot 691 \\
1 \cdot 303 \\
0-003 \\
0-301 \\
0-694 \\
0-014\end{array}$ & $\begin{array}{l}2 \cdot 599 \\
1 \cdot 386 \\
0-012 \\
0-396 \\
0-609 \\
0-011\end{array}$ & $\begin{array}{l}2 \cdot 663 \\
1 \cdot 330 \\
0-001 \\
0-317 \\
0-704 \\
0-016\end{array}$ & $\begin{array}{l}2 \cdot 768 \\
1 \cdot 224 \\
0-006 \\
0-228 \\
0-764 \\
0-018\end{array}$ & $\begin{array}{l}2 \cdot 731 \\
1 \cdot 249 \\
0-019 \\
0-249 \\
0-756 \\
0-020\end{array}$ \\
\hline $\begin{array}{l}\mathrm{Mol} \% \\
\mathrm{Ab} \\
\mathrm{An} \\
\text { Or }\end{array}$ & $\begin{array}{r}91.7 \\
7.6 \\
0.7\end{array}$ & $\begin{array}{r}91 \cdot 8 \\
7 \cdot 4 \\
0.8\end{array}$ & $\begin{array}{r}65 \cdot 3 \\
33 \cdot 7 \\
1 \cdot 1\end{array}$ & $\begin{array}{r}63 \cdot 5 \\
33 \cdot 7 \\
2.9\end{array}$ & $\begin{array}{r}79 \cdot 1 \\
18 \cdot 7 \\
2 \cdot 1\end{array}$ & $\begin{array}{r}78 \cdot 2 \\
20-3 \\
1 \cdot 6\end{array}$ & $\begin{array}{r}68 \cdot 9 \\
29 \cdot 8 \\
1.4\end{array}$ & $\begin{array}{r}59 \cdot 9 \\
39-0 \\
1 \cdot 1\end{array}$ & $\begin{array}{r}67.9 \\
30.6 \\
1.5\end{array}$ & $\begin{array}{r}75.6 \\
22.6 \\
1.8\end{array}$ & $\begin{array}{r}73 \cdot 8 \\
24.3 \\
1.9\end{array}$ \\
\hline
\end{tabular}

* All Fe assumed to be $\mathrm{Fe}^{3+}$.

Table 4

Imenite analyses

\begin{tabular}{|c|c|c|c|c|c|c|}
\hline $\begin{array}{l}\text { Sample } \\
\text { Curcle } \\
\text { Position }\end{array}$ & $\begin{array}{c}7 \\
4 \\
\text { core }\end{array}$ & $\begin{array}{c}7 \\
5 \\
\text { core }\end{array}$ & $\begin{array}{c}9 \\
6 \\
\text { core }\end{array}$ & $\begin{array}{c}9 \\
6 \\
\text { rim }\end{array}$ & $\begin{array}{c}12 \\
3 \\
\text { core }\end{array}$ & $\begin{array}{c}12 \\
4 \\
\text { core }\end{array}$ \\
\hline $\begin{array}{l}\mathrm{SiO}_{2} \\
\mathrm{~T}_{1} \mathrm{O}_{2} \\
\mathrm{Al}_{2} \mathrm{O}_{3} \\
\mathrm{Cr}_{2} \mathrm{O}_{3} \\
\mathrm{Fe}_{2} \mathrm{O}_{3} \\
\mathrm{FeO} \\
\mathrm{MnO} \\
\mathrm{MgO} \\
\mathrm{CaO}\end{array}$ & $\begin{array}{r}0-04 \\
51.81 \\
0-01 \\
0-01 \\
0-50 \\
45.60 \\
0-38 \\
0-45 \\
0-00\end{array}$ & $\begin{array}{r}0-04 \\
51.91 \\
0-00 \\
0-00 \\
0-50 \\
46.20 \\
0.76 \\
0.03 \\
0-03\end{array}$ & $\begin{array}{r}0-02 \\
49.82 \\
0-01 \\
0-00 \\
5 \cdot 20 \\
43.40 \\
1.08 \\
0-02 \\
0-00\end{array}$ & $\begin{array}{r}0-02 \\
48.87 \\
0-01 \\
0-01 \\
6-20 \\
41.60 \\
1.58 \\
0-01 \\
0-72\end{array}$ & $\begin{array}{r}0-00 \\
48 \cdot 66 \\
0-01 \\
0-06 \\
5 \cdot 70 \\
43 \cdot 30 \\
0-35 \\
0.14 \\
0-01\end{array}$ & $\begin{array}{r}0-03 \\
48 \cdot 69 \\
0-02 \\
0-05 \\
5 \cdot 10 \\
42 \cdot 40 \\
0-24 \\
0-54 \\
0-02\end{array}$ \\
\hline Total & $98 \cdot 80$ & $99 \cdot 47$ & 99.55 & $99-02$ & $98-23$ & $97-09$ \\
\hline \multicolumn{7}{|c|}{ Atoms on the basis of 2 cations and 3 oxygens } \\
\hline $\begin{array}{l}\mathrm{Si} \\
\mathrm{Ti} \\
\mathrm{Cr} \\
\mathrm{Fe}^{3+} \\
\mathrm{Fe}^{2+} \\
\mathrm{Mn} \\
\mathrm{Mg} \\
\mathrm{Ca}\end{array}$ & $\begin{array}{c}0-000 \\
0-992 \\
0-000 \\
0-010 \\
0-971 \\
0-008 \\
0-017 \\
0-000\end{array}$ & $\begin{array}{c}0-000 \\
0-991 \\
0-000 \\
0-010 \\
0-980 \\
0-016 \\
0-001 \\
0-001\end{array}$ & $\begin{array}{l}0-000 \\
0-953 \\
0-000 \\
0-100 \\
0-923 \\
0-023 \\
0-001 \\
0-000\end{array}$ & $\begin{array}{l}0-000 \\
0-938 \\
0-000 \\
0-119 \\
0-888 \\
0-034 \\
0-000 \\
0-020\end{array}$ & $\begin{array}{l}0-000 \\
0-942 \\
0-001 \\
0-110 \\
0-932 \\
0-008 \\
0-005 \\
0-000\end{array}$ & $\begin{array}{l}0-000 \\
0-951 \\
0-001 \\
0-100 \\
0-921 \\
0-005 \\
0-021 \\
0-001\end{array}$ \\
\hline
\end{tabular}


average value for biotite touching garnet is $0 \cdot 84$, whereas matrix biotites not in contact with garnet have an $\mathrm{Mg} / \mathrm{Fe}$ ratio of 0.75 . These relationships are typical for biotites from highgrade terranes and indicate partial re-equilibration during cooling.

\section{BAROMETRY}

To obtain estimates of the pressures during the metamorphic history of the Britt domain, various geobarometers were applied to the samples. Core analyses of coexisting minerals were paired in most samples to estimate peak metamorphic conditions. If zoning was present, rim analyses were also used for comparison to limit the retrograde history of the rock. The available diffusion data for garnet summarized by Chakraborty \& Ganguly (1990) and similar data available for pyroxene and plagioclase (cf. Anovitz, 1991) suggest that nearpeak metamorphic $P-T$ conditions may be preserved in rocks regionally metamorphosed to temperatures of $700-750^{\circ} \mathrm{C}$. In samples 8 and 11 the analyses of complexly zoned garnets

TABLE 5

Biotite analyses

\begin{tabular}{|c|c|c|c|c|c|c|c|c|}
\hline $\begin{array}{l}\text { Sample } \\
\text { Position } \\
\text { rel. to Gt }\end{array}$ & near & distant & near & distant & near & distant & distant & included \\
\hline $\mathrm{SiO}_{2}$ & 33.69 & $33-49$ & $33 \cdot 38$ & 33.40 & $34 \cdot 26$ & $34-05$ & 33.90 & $35 \cdot 39$ \\
\hline $\mathrm{TiO}_{2}$ & $4 \cdot 24$ & $4 \cdot 32$ & 277 & 3.90 & $2 \cdot 24$ & 1.96 & $2 \cdot 50$ & 508 \\
\hline $\mathrm{Al}_{2} \mathrm{O}_{3}$ & $18 \cdot 39$ & $18 \cdot 20$ & $18 \cdot 44$ & $18 \cdot 13$ & $19 \cdot 29$ & $19 \cdot 81$ & $19 \cdot 22$ & $18 \cdot 34$ \\
\hline $\mathrm{Cr}_{2} \mathrm{O}_{3}$ & $0-01$ & $0-00$ & $0-02$ & $0-01$ & $0-00$ & $0-02$ & $0-05$ & $0-00$ \\
\hline $\mathrm{FeO}$ & 19.93 & $20 \cdot 34$ & $20-33$ & $21 \cdot 16$ & $21 \cdot 59$ & $21 \cdot 28$ & $21 \cdot 25$ & 13.86 \\
\hline $\mathrm{MnO}$ & $0-00$ & $0-10$ & $0-03$ & $0-06$ & $0-50$ & $0-10$ & $0-05$ & $0-01$ \\
\hline $\mathrm{MgO}$ & $8 \cdot 22$ & $8 \cdot 13$ & 8.95 & $7 \cdot 83$ & $7 \cdot 88$ & $8 \cdot 44$ & $8 \cdot 76$ & $12 \cdot 04$ \\
\hline $\mathrm{BaO}$ & $0-00$ & $0-00$ & 000 & $0-05$ & $0-08$ & $0-03$ & $0-02$ & $0-88$ \\
\hline $\mathrm{CaO}$ & $0-00$ & $0-00$ & $0-01$ & $0-00$ & $0-05$ & $0-05$ & $0-03$ & $0-00$ \\
\hline $\mathrm{Na}_{2} \mathrm{O}$ & 0.10 & 0.13 & $0-08$ & $0-04$ & 0.12 & $0-09$ & $0-10$ & $0-08$ \\
\hline $\mathrm{K}_{2} \mathrm{O}$ & $9 \cdot 73$ & $9 \cdot 85$ & $9 \cdot 78$ & $9 \cdot 73$ & $9-07$ & $9 \cdot 55$ & $9-00$ & $9 \cdot 65$ \\
\hline $\mathrm{H}_{2} \mathrm{O}$ & 2.45 & $2 \cdot 50$ & $2 \cdot 53$ & $2 \cdot 35$ & 2.97 & 302 & $3 \cdot 18$ & $2 \cdot 31$ \\
\hline$F^{2}$ & $0-19$ & $0-14$ & $0-87$ & 069 & $0-28$ & $0-32$ & 018 & 0.46 \\
\hline $\mathrm{Cl}$ & $0-00$ & $0-00$ & $0-09$ & $0-09$ & $0-00$ & $0-01$ & $0-01$ & $0-14$ \\
\hline $\mathrm{O}=\mathrm{F}$ & $0-08$ & $0-06$ & 0.37 & $0-29$ & 0.12 & $0-11$ & $0-07$ & 0.19 \\
\hline $\mathrm{O}=\mathrm{Cl}$ & $0-00$ & 000 & $0-02$ & $0-02$ & $0-00$ & $0-00$ & $0-00$ & $0-03$ \\
\hline Total & 96.87 & $97 \cdot 14$ & 96.89 & $97 \cdot 13$ & $98 \cdot 21$ & 98.62 & $98 \cdot 18$ & 98.02 \\
\hline \multicolumn{9}{|c|}{ Atoms on the basis of $14(I V+V I)$ cations } \\
\hline$S_{1}$ & $5 \cdot 392$ & $5 \cdot 360$ & 5.335 & $5 \cdot 365$ & $5 \cdot 375$ & $5 \cdot 332$ & $5 \cdot 312$ & $5 \cdot 482$ \\
\hline$A l^{I V}$ & $2 \cdot 608$ & $2 \cdot 640$ & 2.665 & 2.635 & 2.625 & 2.668 & 2.688 & $2 \cdot 518$ \\
\hline $\mathrm{Al}^{\mathrm{VI}}$ & $0-862$ & $0-794$ & $0-810$ & 0.798 & 0.943 & $0-989$ & $0-863$ & $0-831$ \\
\hline $\mathrm{Ti}$ & $0-510$ & $0-520$ & $0-333$ & $0-471$ & $0-264$ & $0-231$ & $0-295$ & $0-592$ \\
\hline $\mathrm{Cr}$ & $0-001$ & $0-000$ & $0-003$ & $0-001$ & $0-000$ & $0-002$ & $0-006$ & $0-000$ \\
\hline $\mathrm{Fe}$ & $2 \cdot 668$ & $2 \cdot 723$ & $2 \cdot 718$ & $2 \cdot 842$ & 2.833 & $2 \cdot 787$ & $2 \cdot 785$ & 1.795 \\
\hline$M n$ & $0-000$ & $0-024$ & $0-007$ & $0-014$ & $0-117$ & $0-023$ & $0-012$ & $0-002$ \\
\hline $\mathrm{Mg}$ & 1.961 & 1.939 & $2 \cdot 132$ & 1.874 & $1 \cdot 843$ & 1.970 & $2-046$ & $2 \cdot 779$ \\
\hline $\mathrm{Ba}$ & $0-000$ & $0-000$ & $0-000$ & $0-003$ & $0-005$ & $0-002$ & $0-001$ & $0-053$ \\
\hline $\mathrm{Ca}$ & $0-000$ & $0-000$ & $0-002$ & $0-000$ & $0-008$ & $0-008$ & $0-005$ & $0-000$ \\
\hline $\mathrm{Na}$ & $0-031$ & $0-040$ & $0-025$ & $0-012$ & $0-037$ & $0-027$ & $0-030$ & $0-024$ \\
\hline $\mathrm{K}$ & 1.987 & 2011 & 1.994 & 1.994 & 1.816 & 1.908 & 1.799 & 1.907 \\
\hline 0 & $21 \cdot 288$ & $21 \cdot 260$ & $20-838$ & $21 \cdot 107$ & 20753 & 20687 & $20-587$ & $21 \cdot 351$ \\
\hline $\mathrm{OH}$ & 2.616 & 2.669 & 2.698 & 2.518 & $3 \cdot 108$ & $3 \cdot 152$ & $3 \cdot 321$ & $2 \cdot 387$ \\
\hline$F$ & $0-096$ & $0-071$ & $0-440$ & $0-351$ & $0-139$ & $0-158$ & $0-089$ & 0.225 \\
\hline $\mathrm{Cl}$ & $0-000$ & $0-000$ & $0-024$ & $0-024$ & $0-000$ & $0-003$ & $0-003$ & $0-037$ \\
\hline
\end{tabular}


TABLE 5 (Continued)

\begin{tabular}{|c|c|c|c|c|c|c|}
\hline $\begin{array}{l}\text { Sample } \\
\text { Position } \\
\text { rel. to Gt }\end{array}$ & touching & distant & $\begin{array}{c}9 \\
\text { distant }\end{array}$ & near & $\begin{array}{c}11 \\
\text { near }\end{array}$ & $\begin{array}{c}I I \\
\text { distant }\end{array}$ \\
\hline $\mathrm{SiO}_{2}$ & $34-01$ & $33 \cdot 60$ & 34.53 & 34.92 & 34.44 & $34 \cdot 14$ \\
\hline $\mathrm{TiO}_{2}$ & $3 \cdot 40$ & $4 \cdot 21$ & $4 \cdot 47$ & $4 \cdot 28$ & $4 \cdot 32$ & 4.02 \\
\hline $\mathrm{Al}_{2} \mathrm{O}_{3}$ & 19.03 & 18.29 & 13.71 & 13.60 & 18.18 & 18.01 \\
\hline $\mathrm{Cr}_{2} \mathrm{O}_{3}$ & $0-04$ & $0-06$ & $0-03$ & $0-01$ & $0-27$ & $0-21$ \\
\hline $\mathrm{FeO}$ & $19 \cdot 86$ & 19.91 & $28 \cdot 65$ & $29 \cdot 35$ & $18 \cdot 75$ & $19 \cdot 26$ \\
\hline $\mathrm{MnO}$ & $0-06$ & $0-06$ & $0-21$ & $0-21$ & $0-00$ & $0-08$ \\
\hline $\mathrm{MgO}$ & $9-00$ & $8 \cdot 48$ & $5 \cdot 19$ & 4.75 & $9 \cdot 16$ & $9 \cdot 15$ \\
\hline $\mathrm{BaO}$ & $0-00$ & $0-00$ & 0.21 & 0.18 & $0-00$ & $0-00$ \\
\hline $\mathrm{CaO}$ & $0-09$ & $0-06$ & $0-04$ & $0-00$ & $0-01$ & $0-02$ \\
\hline $\mathrm{Na}_{2} \mathrm{O}$ & 016 & $0-05$ & $0-04$ & $0-02$ & $0-04$ & $0-07$ \\
\hline $\mathrm{K}_{2} \mathrm{O}$ & $9 \cdot 29$ & 9.93 & $9 \cdot 49$ & $9 \cdot 49$ & 9.86 & 9.66 \\
\hline $\mathrm{H}_{2} \mathrm{O}$ & $2 \cdot 66$ & $2 \cdot 41$ & $2 \cdot 53$ & $2 \cdot 41$ & $2 \cdot 21$ & 2.43 \\
\hline$F^{2}$ & 0.51 & $0-39$ & 0.57 & 0.73 & 0.62 & 0.46 \\
\hline $\mathrm{Cl}$ & $0-16$ & 0.12 & $0-07$ & $0-07$ & $0-20$ & 0.24 \\
\hline $\mathrm{O}=\mathrm{F}$ & 0.21 & 0.16 & $0-24$ & $0-31$ & $0-26$ & $0-19$ \\
\hline $\mathrm{O}=\mathrm{Cl}$ & $0-04$ & $0-03$ & $0-02$ & $0-02$ & $0-04$ & $0-05$ \\
\hline Total & 98.02 & 97.38 & 99.48 & 99.69 & 97.76 & $97 \cdot 51$ \\
\hline \multicolumn{7}{|c|}{ Atoms on the basis of $14(I V+V I)$ cations } \\
\hline$S_{1}$ & $5 \cdot 343$ & $5 \cdot 363$ & 5.617 & 5.677 & $5 \cdot 451$ & $5 \cdot 415$ \\
\hline $\mathrm{All}^{\mathrm{IV}}$ & $2 \cdot 657$ & 2.637 & $2 \cdot 383$ & $2 \cdot 323$ & $2 \cdot 549$ & $2 \cdot 585$ \\
\hline $\mathrm{Al}^{\mathrm{vI}}$ & $0-868$ & $0-805$ & $0-246$ & $0-284$ & $0-843$ & $0-783$ \\
\hline $\mathrm{Ti}$ & $0-402$ & $0-505$ & $0-547$ & $0-523$ & 0.514 & $0-480$ \\
\hline $\mathrm{Cr}$ & $0-005$ & $0-008$ & $0-004$ & $0-001$ & $0-034$ & $0-026$ \\
\hline $\mathrm{Fe}$ & 2.609 & $2 \cdot 658$ & 3.898 & 3.991 & $2 \cdot 482$ & $2 \cdot 555$ \\
\hline Mn & $0-014$ & $0-014$ & $0-051$ & $0-051$ & $0-000$ & $0-019$ \\
\hline $\mathrm{Mg}$ & $2 \cdot 107$ & 2017 & $1 \cdot 258$ & $1 \cdot 151$ & $2 \cdot 161$ & $2 \cdot 163$ \\
\hline $\mathrm{Ba}$ & $0-000$ & $0-000$ & $0-013$ & $0-011$ & $0-000$ & $0-000$ \\
\hline $\mathrm{Ca}$ & $0-015$ & $0-010$ & $0-007$ & $0-000$ & $0-002$ & $0-003$ \\
\hline $\mathrm{Na}$ & $0-049$ & $0-015$ & $0-013$ & $0-006$ & $0-012$ & $0-022$ \\
\hline K & 1.862 & 2.022 & 1.970 & 1.968 & 1.991 & 1.995 \\
\hline 0 & $20-916$ & $21 \cdot 205$ & $20-942$ & $20-992$ & $21 \cdot 303$ & $21 \cdot 133$ \\
\hline $\mathrm{OH}$ & $2 \cdot 788$ & $2 \cdot 566$ & $2 \cdot 745$ & $2 \cdot 614$ & $2 \cdot 333$ & 2.571 \\
\hline $\mathbf{F}$ & 0.253 & 0.197 & $0-293$ & $0-375$ & $0-310$ & $0-231$ \\
\hline $\mathrm{Cl}$ & $0-043$ & $0-032$ & $0-019$ & $0-019$ & $0-054$ & $0-065$ \\
\hline
\end{tabular}

B1otites were normalized assuming $(\mathrm{Mg}+\mathrm{Fe}+\mathrm{Ti}+\mathrm{Mn}+\mathrm{Zn}+\mathrm{Al}+\mathrm{Si})=14$ and assuming all $\mathrm{Fe}$ is $\mathrm{Fe}^{2+} . \mathrm{H}$ content has been calculated from charge balance assuming the occupancy of the $\mathrm{OH}$ site is four.

with the highest and lowest grossular contents were both paired with unzoned matrix plagioclase. Pressures are presented in Table 9 for three reference temperatures, 650, 700, and $750^{\circ} \mathrm{C}$, which are considered to be reasonable estimates of regional conditions based on thermometry from the Britt domain by Anovitz \& Essene (1990) and the mineral assemblages described in this paper. For comparison, pressures calculated at $700{ }^{\circ} \mathrm{C}$ were used. Methods used to constrain temperature in the samples are discussed below.

Metapelitic and tonalitic rocks

The reaction

$$
\begin{aligned}
& \text { anorthite }=\text { grossular }+ \text { kyanite }+ \text { quartz } \\
& 3 \mathrm{CaAl}_{2} \mathrm{Si}_{2} \mathrm{O}_{8}=\mathrm{Ca}_{3} \mathrm{Al}_{2} \mathrm{Si}_{3} \mathrm{O}_{12}+2 \mathrm{Al}_{2} \mathrm{SiO}_{5}+\mathrm{SiO}_{2}
\end{aligned}
$$


TABLE 6

Spinel analyses

\begin{tabular}{|c|c|c|c|c|c|c|c|c|}
\hline $\begin{array}{l}\text { Sample } \\
\text { Circle } \\
\text { Position } \\
\text { Touching }\end{array}$ & $\begin{array}{c}7 \\
6 \\
\text { core } \\
S t\end{array}$ & $\begin{array}{c}7 \\
6 \\
\text { rim } \\
S t\end{array}$ & $\begin{array}{c}7 \\
5 \\
\text { core } \\
G t\end{array}$ & $\begin{array}{c}7 \\
5 \\
\text { rim } \\
G t\end{array}$ & $\begin{array}{c}7 \\
2 \\
\text { core } \\
-\end{array}$ & $\begin{array}{c}7 \\
2 \\
\text { rim } \\
-\end{array}$ & $\begin{array}{c}7 \\
3 \\
\text { rim } \\
S t\end{array}$ & $\begin{array}{c}7 \\
3 \\
\text { core } \\
S t\end{array}$ \\
\hline $\begin{array}{l}\mathrm{SiO}_{2} \\
\mathrm{TiO}_{2} \\
\mathrm{Al}_{2} \mathrm{O}_{3} \\
\mathrm{Cr}_{2} \mathrm{O}_{3} \\
\mathrm{Fe}_{2} \mathrm{O}_{3} \\
\mathrm{FeO} \\
\mathrm{MnO} \\
\mathrm{MgO} \\
\mathrm{ZnO}\end{array}$ & $\begin{array}{r}0-03 \\
0-00 \\
56.95 \\
0-00 \\
0.44 \\
9.86 \\
0-08 \\
1.49 \\
31.50\end{array}$ & $\begin{array}{r}0-01 \\
0-00 \\
56.13 \\
0-00 \\
1.14 \\
8.83 \\
0.08 \\
1.43 \\
32.44\end{array}$ & $\begin{array}{r}0-03 \\
0-00 \\
57-00 \\
0-00 \\
0-23 \\
10-02 \\
0-04 \\
1.59 \\
31-09\end{array}$ & $\begin{array}{r}0.00 \\
0-01 \\
56 \cdot 16 \\
0-00 \\
0.59 \\
9 \cdot 46 \\
0.06 \\
1.46 \\
32.21\end{array}$ & $\begin{array}{r}0-04 \\
0-01 \\
58 \cdot 11 \\
0-00 \\
0.19 \\
11 \cdot 26 \\
0-08 \\
2.46 \\
28 \cdot 78\end{array}$ & $\begin{array}{r}0-04 \\
0-00 \\
58.65 \\
0-00 \\
0-00 \\
11 \cdot 27 \\
0-05 \\
2.51 \\
28.90\end{array}$ & $\begin{array}{r}0-03 \\
0-02 \\
57.64 \\
0-00 \\
0-01 \\
11.68 \\
0-09 \\
2.36 \\
28.06\end{array}$ & $\begin{array}{r}0-04 \\
0-00 \\
57.44 \\
0-00 \\
0-07 \\
11.78 \\
0-07 \\
2.38 \\
27.74\end{array}$ \\
\hline Total & $100-35$ & $100-06$ & $100-00$ & $100-95$ & $100-93$ & $101 \cdot 42$ & 99.89 & $99 \cdot 52$ \\
\hline \multicolumn{9}{|c|}{ Atoms on the basis of 3 cations and 4 oxygens } \\
\hline $\begin{array}{l}\mathrm{Si} \\
\mathrm{Ti} \\
\mathrm{Al} \\
\mathrm{Cr} \\
\mathrm{Fe}^{3+} \\
\mathrm{Fe}^{3+} \\
\mathrm{Mn}^{2+} \\
\mathrm{Mg} \\
\mathrm{Zn}\end{array}$ & $\begin{array}{l}0-001 \\
0-000 \\
1.988 \\
0-000 \\
0-010 \\
0-244 \\
0-002 \\
0-066 \\
0-689\end{array}$ & $\begin{array}{l}0-000 \\
0-000 \\
1.974 \\
0-000 \\
0-026 \\
0-220 \\
0-002 \\
0-064 \\
0-714\end{array}$ & $\begin{array}{l}0-001 \\
0-000 \\
1.993 \\
0-000 \\
0-005 \\
0-249 \\
0-001 \\
0-070 \\
0-681\end{array}$ & $\begin{array}{l}0-000 \\
0-000 \\
1.987 \\
0-000 \\
0-013 \\
0-233 \\
0-001 \\
0-064 \\
0-701\end{array}$ & $\begin{array}{l}0-001 \\
0-000 \\
1.993 \\
0-000 \\
0-004 \\
0-274 \\
0-002 \\
0-107 \\
0-618\end{array}$ & $\begin{array}{l}0-001 \\
0-000 \\
2-000 \\
0-000 \\
0-000 \\
0-273 \\
0-001 \\
0-108 \\
0-617\end{array}$ & $\begin{array}{l}0-001 \\
0-000 \\
1.997 \\
0-000 \\
0-000 \\
0-287 \\
0-002 \\
0-103 \\
0-609\end{array}$ & $\begin{array}{l}0-001 \\
0-000 \\
1.997 \\
0-000 \\
0-002 \\
0-290 \\
0-002 \\
0-105 \\
0-604\end{array}$ \\
\hline
\end{tabular}

was applied to all metapelites using the experimental calibration of Koziol \& Newton (1988) and thermodynamic data from Anovitz \& Essene (1987). The garnet activity model of Ganguly \& Saxena (1984) as modified by Anovitz \& Essene (1987) was used. The activity of $\mathrm{CaAl}_{2} \mathrm{Si}_{2} \mathrm{O}_{8}$ in plagioclase was calculated with the model of Fuhrman \& Lindsley (1988).

The pressures obtained with the GASP barometer for metapelitic samples 8 and 11 show a large difference between the high- and low-Ca areas of the garnets. The pressures obtained using analyses from $\mathrm{Ca}$-rich portions of the grains are 10.8 and $11.2 \mathrm{~kb}$ for samples 8 and 11 respectively, whereas analyses from low-grossular regions of the grains yield pressures of 6.2 and $5.4 \mathrm{~kb}$ (Table 9). Increasing the reference temperature by $50^{\circ} \mathrm{C}$ raises the pressures by $1 \mathrm{~kb}$, but the difference does not change. Pressures obtained for the other three metapelites from the Britt domain span a 5-kb range and are intermediate to those given by the complexly zoned garnets in samples 8 and 11 . Rim values are $\sim 0.7 \mathrm{~kb}$ lower than the corresponding core pressure in sample $12,0.4 \mathrm{~kb}$ lower in sample 6 and $\sim 1.5 \mathrm{~kb}$ lower in sample 7.

Pressures for the three metapelitic samples from the Parry Sound Shear Zone are similar to lower values obtained from the samples within the Britt domain. Calculations with rim analyses yield pressures that are $\sim 0.3 \mathrm{~kb}$ lower than core values in sample 4 , nearly $1 \mathrm{~kb}$ lower in sample 5 and nearly $2 \mathrm{~kb}$ lower in sample 1 .

In all of the metapelites except sample 5, the full GASP assemblage is present. In this sample, kyanite was found as inclusions in garnet but not in the matrix. Pressure estimates for this sample should, therefore, be considered an upper limit. 
TABLE 7

Staurolite analyses for sample Sh 88-2a

\begin{tabular}{|c|c|c|c|c|c|c|c|c|c|}
\hline $\begin{array}{l}\text { Circle } \\
\text { Position } \\
\text { Touching }\end{array}$ & $\begin{array}{c}2 \\
\text { core } \\
G t\end{array}$ & $\begin{array}{c}2 \\
\text { core } \\
G t\end{array}$ & $\underset{\mathrm{rim}}{2}$ & $\begin{array}{c}3 \\
\text { core } \\
S p l\end{array}$ & $\begin{array}{c}3 \\
\text { rim } \\
\text { Spl }\end{array}$ & $\begin{array}{c}5 \\
\text { core } \\
-\end{array}$ & $\begin{array}{c}5 \\
\text { rim } \\
-\end{array}$ & $\begin{array}{c}6 \\
\text { core } \\
-\end{array}$ & $\begin{array}{c}6 \\
\text { rim } \\
-\end{array}$ \\
\hline $\begin{array}{l}\mathrm{SiO}_{2} \\
\mathrm{TiO}_{2} \\
\mathrm{Al}_{2} \mathrm{O}_{3} \\
\mathrm{Fe}_{2} \mathrm{O}_{3} \\
\mathrm{FeO} \\
\mathrm{MnO} \\
\mathrm{MgO} \\
\mathrm{ZnO} \\
\mathrm{F} \\
\mathrm{Cl} \\
\mathrm{H}_{2} \mathrm{O}\end{array}$ & $\begin{array}{r}26 \cdot 78 \\
0.71 \\
54 \cdot 17 \\
1.18 \\
9.39 \\
0.08 \\
1.88 \\
4.62 \\
0.00 \\
0-00 \\
1.36\end{array}$ & $\begin{array}{r}26.55 \\
1.09 \\
54.13 \\
1.18 \\
9.57 \\
0.11 \\
1.81 \\
4.55 \\
0-00 \\
000 \\
1.19\end{array}$ & $\begin{array}{r}26 \cdot 83 \\
1.05 \\
53 \cdot 98 \\
1 \cdot 18 \\
9 \cdot 64 \\
0.07 \\
1.90 \\
4 \cdot 13 \\
0.00 \\
0.00 \\
1 \cdot 14\end{array}$ & $\begin{array}{r}26 \cdot 64 \\
1 \cdot 12 \\
54.08 \\
1 \cdot 18 \\
10.30 \\
0.14 \\
1.80 \\
3.86 \\
0-00 \\
0-00 \\
1 \cdot 13\end{array}$ & $\begin{array}{r}27.05 \\
1.09 \\
53 \cdot 50 \\
1.17 \\
10.35 \\
0.12 \\
1.88 \\
3.94 \\
0-00 \\
0-00 \\
0-71\end{array}$ & $\begin{array}{r}26 \cdot 31 \\
0.71 \\
53 \cdot 92 \\
1 \cdot 17 \\
9 \cdot 24 \\
0.13 \\
1 \cdot 51 \\
5 \cdot 10 \\
0-00 \\
0-00 \\
1 \cdot 43\end{array}$ & $\begin{array}{r}26.97 \\
0.68 \\
53 \cdot 70 \\
1 \cdot 16 \\
9 \cdot 40 \\
0.14 \\
1.53 \\
4.79 \\
0-00 \\
0-00 \\
1.38\end{array}$ & $\begin{array}{r}26.22 \\
0.42 \\
54.95 \\
1.18 \\
9.57 \\
0.11 \\
1.28 \\
5.31 \\
0-00 \\
0-00 \\
1.66\end{array}$ & $\begin{array}{r}26 \cdot 10 \\
0.32 \\
53 \cdot 72 \\
1.16 \\
9 \cdot 39 \\
0.13 \\
1.30 \\
5 \cdot 39 \\
0-00 \\
0.00 \\
1.58\end{array}$ \\
\hline Total & $100-17$ & $100-18$ & $99 \cdot 92$ & $100-25$ & 99.81 & 99.52 & 99.75 & $100-72$ & $99-09$ \\
\hline \multicolumn{10}{|c|}{ Atoms based on $25.53(V I+I V)$ cations $^{*}$} \\
\hline $\begin{array}{l}\mathrm{Si} \\
\mathrm{Ti} \\
\mathrm{Al} \\
\mathrm{Fe}^{3+} \\
\mathrm{Fe}^{2+} \\
\mathrm{Mn} \\
\mathrm{Mg} \\
\mathrm{Zn} \\
\mathrm{F} \\
\mathrm{Cl} \\
\mathrm{OH}\end{array}$ & $\begin{array}{r}7.54 \\
0-15 \\
17.99 \\
0-25 \\
2.21 \\
0-02 \\
0-79 \\
0-96 \\
0-00 \\
0-00 \\
2.56\end{array}$ & $\begin{array}{r}7.50 \\
0-23 \\
18.03 \\
0-25 \\
2.26 \\
0-03 \\
0-76 \\
0-95 \\
0-00 \\
0-00 \\
2.24\end{array}$ & $\begin{array}{r}7.58 \\
0.22 \\
17.96 \\
0.25 \\
2.27 \\
0-02 \\
0-80 \\
0.94 \\
0-00 \\
0-00 \\
2.14\end{array}$ & $\begin{array}{r}7.53 \\
0.24 \\
18.00 \\
0.25 \\
2.43 \\
0.03 \\
0.76 \\
0.80 \\
0-00 \\
0-00 \\
2.13\end{array}$ & $\begin{array}{r}7.66 \\
0.23 \\
17.87 \\
0.25 \\
2.45 \\
0-29 \\
0-80 \\
0.82 \\
0-00 \\
0-00 \\
1.34\end{array}$ & $\begin{array}{r}7.47 \\
0.15 \\
18.05 \\
0.25 \\
2.20 \\
0-03 \\
0.64 \\
1.07 \\
0.00 \\
0-00 \\
2.71\end{array}$ & $\begin{array}{r}7.63 \\
0-14 \\
17.90 \\
0-25 \\
2.25 \\
0-03 \\
0.65 \\
1.00 \\
0-00 \\
0-00 \\
2.62\end{array}$ & $\begin{array}{r}7 \cdot 36 \\
0-09 \\
18 \cdot 17 \\
0.25 \\
2.25 \\
0-03 \\
0-54 \\
1 \cdot 10 \\
0-00 \\
0-00 \\
3 \cdot 10\end{array}$ & $\begin{array}{r}7.45 \\
0-07 \\
18-08 \\
0-25 \\
2.24 \\
0-03 \\
0-55 \\
1.14 \\
0-00 \\
0-00 \\
3-02\end{array}$ \\
\hline
\end{tabular}

* Formulae were calculated according to the recommendations of Holdaway et al. (1986).

The barometer

$$
\begin{aligned}
& \text { anorthite }+ \text { ilmenite }+ \text { quartz }=\text { grossular }+ \text { almandine }+ \text { rutile } \\
& 3 \mathrm{CaAl}_{2} \mathrm{Si}_{2} \mathrm{O}_{8}+6 \mathrm{FeTiO}_{3}+3 \mathrm{SiO}_{2}=\mathrm{Ca}_{3} \mathrm{Al}_{2} \mathrm{Si}_{3} \mathrm{O}_{12}+2 \mathrm{Fe}_{3} \mathrm{Al}_{2} \mathrm{Si}_{3} \mathrm{O}_{12}+6 \mathrm{TiO}_{2}
\end{aligned}
$$

(GRIPS)

from Bohlen \& Liotta (1986) was applied to most of the metapelites and one tonalitic sample. An ideal model was used for the activity of $\mathrm{FeTiO}_{3}$ in ilmenite, and the activity of $\mathrm{TiO}_{2}$ was taken as unity. Samples 5, 7, 9, and 12 contain the full GRIPS assemblage. Sample 9 yields the highest GRIPS pressures, slightly higher than the maximum pressure obtained for sample 11. Because of a lack of primary ilmenite, pressures obtained for samples 1, 5, 6, 8, and 11 must be considered lower limits. Agreement between the GASP and GRIPS barometers is within $1.4 \mathrm{~kb}$ when the two assemblages are fully buffered.

The GASP and GRIPS barometers involve the grossular content of garnet, which is generally low in metapelitic rocks. Therefore, small uncertainties in the measurement of the Ca content may result in large uncertainties in calculated pressures. However, sample 9 has a high grossular content and contains the full GRIPS assemblage. Application of the GRIPS barometer to this rock yields a pressure of $11.9 \mathrm{~kb}$ at $700^{\circ} \mathrm{C}$, i.e., $<1 \mathrm{~kb}$ higher than the GASP pressures from the high grossular areas of the complexly zoned garnets in sample 8 . 
TABle 8

Clinopyroxene analyses

\begin{tabular}{|c|c|c|c|c|c|c|}
\hline $\begin{array}{l}\text { Sample } \\
\text { Position }\end{array}$ & $\begin{array}{c}10 \\
\text { core }\end{array}$ & $\begin{array}{c}10 \\
\mathrm{rim}\end{array}$ & $\begin{array}{c}2 \\
\text { core }\end{array}$ & $\underset{\text { rim }}{2}$ & $\begin{array}{c}3 \\
\text { core }\end{array}$ & $\begin{array}{c}3 \\
\text { rim }\end{array}$ \\
\hline $\mathrm{SiO}_{2}$ & 5305 & 53.07 & $53 \cdot 46$ & 53.55 & $54 \cdot 79$ & $53 \cdot 37$ \\
\hline $\mathrm{TiO}_{2}$ & $0-24$ & 0.14 & $0-05$ & $0-08$ & $0-13$ & $0-12$ \\
\hline $\mathrm{Al}_{2} \mathrm{O}_{3}$ & $2 \cdot 23$ & 201 & $1-00$ & $1 \cdot 11$ & 1.65 & $1 \cdot 38$ \\
\hline $\mathrm{Cr}_{2} \mathrm{O}_{3}$ & $0-02$ & $0-03$ & $0-00$ & $0-01$ & $0-02$ & $0-06$ \\
\hline $\mathrm{Fe}_{2} \mathrm{O}_{3}$ & $0-02$ & $0-03$ & $0-00$ & $0-01$ & $0-02$ & $0-00$ \\
\hline $\mathrm{FeO}$ & $7 \cdot 44$ & $7 \cdot 28$ & $9 \cdot 59$ & $9 \cdot 13$ & $6 \cdot 76$ & $6-03$ \\
\hline $\mathrm{MnO}$ & $0-10$ & 0.13 & $0-09$ & 0.13 & $0-08$ & $0-07$ \\
\hline $\mathrm{MgO}$ & 13.56 & 13.61 & 13.02 & $12 \cdot 64$ & $14 \cdot 22$ & $14-33$ \\
\hline $\mathrm{CaO}$ & $22 \cdot 72$ & $22 \cdot 52$ & $22 \cdot 80$ & $22 \cdot 71$ & 22.99 & $23 \cdot 09$ \\
\hline $\mathrm{Na}_{2} \mathrm{O}$ & 0.56 & 0.39 & 0.37 & 0.42 & $0-42$ & $0-32$ \\
\hline Total & 99.94 & $99 \cdot 21$ & $100-38$ & $99 \cdot 79$ & 101.08 & $98 \cdot 77$ \\
\hline \multicolumn{7}{|c|}{ Atoms on the basis of 4 cations and 6 oxygens } \\
\hline $\mathrm{Si}$ & 1.978 & 1.992 & 1.999 & 2016 & 2014 & 2.002 \\
\hline Al & $0-098$ & $0-089$ & $0-044$ & $0-049$ & $0-072$ & $0-061$ \\
\hline $\mathrm{Ti}$ & $0-007$ & $0-004$ & $0-001$ & $0-002$ & $0-004$ & $0-003$ \\
\hline $\mathrm{Cr}$ & $0-001$ & $0-001$ & $0-000$ & $0-000$ & $0-001$ & $0-002$ \\
\hline $\mathrm{Fe}^{3+}$ & $0-001$ & $0-001$ & $0-000$ & $0-000$ & $0-001$ & $0-000$ \\
\hline $\mathrm{Fe}^{2+}$ & $0-232$ & $0-228$ & 0.300 & 0.287 & $0-208$ & 0.189 \\
\hline $\mathbf{M n}$ & $0-003$ & $0-004$ & $0-003$ & $0-004$ & $0-002$ & $0-002$ \\
\hline Mg & 0.753 & 0.761 & 0.726 & 0709 & 0.779 & $0-801$ \\
\hline $\mathrm{Ca}$ & $0-908$ & 0.906 & $0-914$ & 0916 & $0-906$ & $0-928$ \\
\hline $\mathrm{Na}$ & $0-040$ & $0-028$ & $0-027$ & $0-031$ & $0-030$ & $0-023$ \\
\hline
\end{tabular}

Table 9

Pressures

\begin{tabular}{|c|c|c|c|c|c|c|c|}
\hline Sample & Circle & $\begin{array}{l}\text { Data } \\
\text { type }\end{array}$ & Barometer & $T_{\text {ref }}$ & $\mathrm{P}(A \& E)$ & $\mathrm{P}($ Ber $)$ & Limit \\
\hline 1 & $\begin{array}{l}1 \\
1 \\
1 \\
1 \\
1 \\
1 \\
2 \\
2 \\
2 \\
2 \\
2 \\
2 \\
2 \\
2 \\
2 \\
2 \\
2 \\
2\end{array}$ & $\begin{array}{l}\text { cores } \\
\text { cores } \\
\text { cores } \\
\text { cores } \\
\text { cores } \\
\text { cores } \\
\text { cores } \\
\text { cores } \\
\text { cores } \\
\text { rims } \\
\text { rims } \\
\text { rims } \\
\text { cores } \\
\text { cores } \\
\text { cores } \\
\text { rims } \\
\text { rims } \\
\text { rims }\end{array}$ & $\begin{array}{l}\text { GASP } \\
\text { GASP } \\
\text { GASP } \\
\text { GRIPS } \\
\text { GRIPS } \\
\text { GRIPS } \\
\text { GASP } \\
\text { GASP } \\
\text { GASP } \\
\text { GASP } \\
\text { GASP } \\
\text { GASP } \\
\text { GRIPS } \\
\text { GRIPS } \\
\text { GRIPS } \\
\text { GRIPS } \\
\text { GRIPS } \\
\text { GRIPS }\end{array}$ & $\begin{array}{l}650 \\
700 \\
750 \\
650 \\
700 \\
750 \\
650 \\
700 \\
750 \\
650 \\
700 \\
750 \\
650 \\
700 \\
750 \\
650 \\
700 \\
750\end{array}$ & $\begin{array}{l}7 \cdot 2 \\
8 \cdot 0 \\
8 \cdot 8 \\
6 \cdot 9 \\
7 \cdot 6 \\
8 \cdot 3 \\
7 \cdot 0 \\
7 \cdot 8 \\
8 \cdot 6 \\
5 \cdot 4 \\
6 \cdot 1 \\
6 \cdot 9 \\
6 \cdot 8 \\
7 \cdot 5 \\
8 \cdot 2 \\
6 \cdot 1 \\
6.7 \\
7 \cdot 4\end{array}$ & $\begin{array}{l}7.8 \\
8.6 \\
9.6\end{array}$ & $\begin{array}{l}\text { lower } \\
\text { lower } \\
\text { lower } \\
\text { lower } \\
\text { lower } \\
\text { lower }\end{array}$ \\
\hline 2 & $\begin{array}{l}1 \\
1 \\
1 \\
1\end{array}$ & $\begin{array}{l}\text { cores } \\
\text { cores } \\
\text { cores } \\
\text { rims }\end{array}$ & $\begin{array}{l}\text { GAHS } \\
\text { GAHS } \\
\text { GAHS } \\
\text { GAHS }\end{array}$ & $\begin{array}{l}650 \\
700 \\
750 \\
650\end{array}$ & $\begin{array}{r}8 \cdot 2 \\
91 \\
10-0 \\
8 \cdot 4\end{array}$ & $\begin{array}{l}7.5 \\
8 \cdot 6 \\
9.6 \\
7.6\end{array}$ & \\
\hline
\end{tabular}


TABLE 9 (Continued)

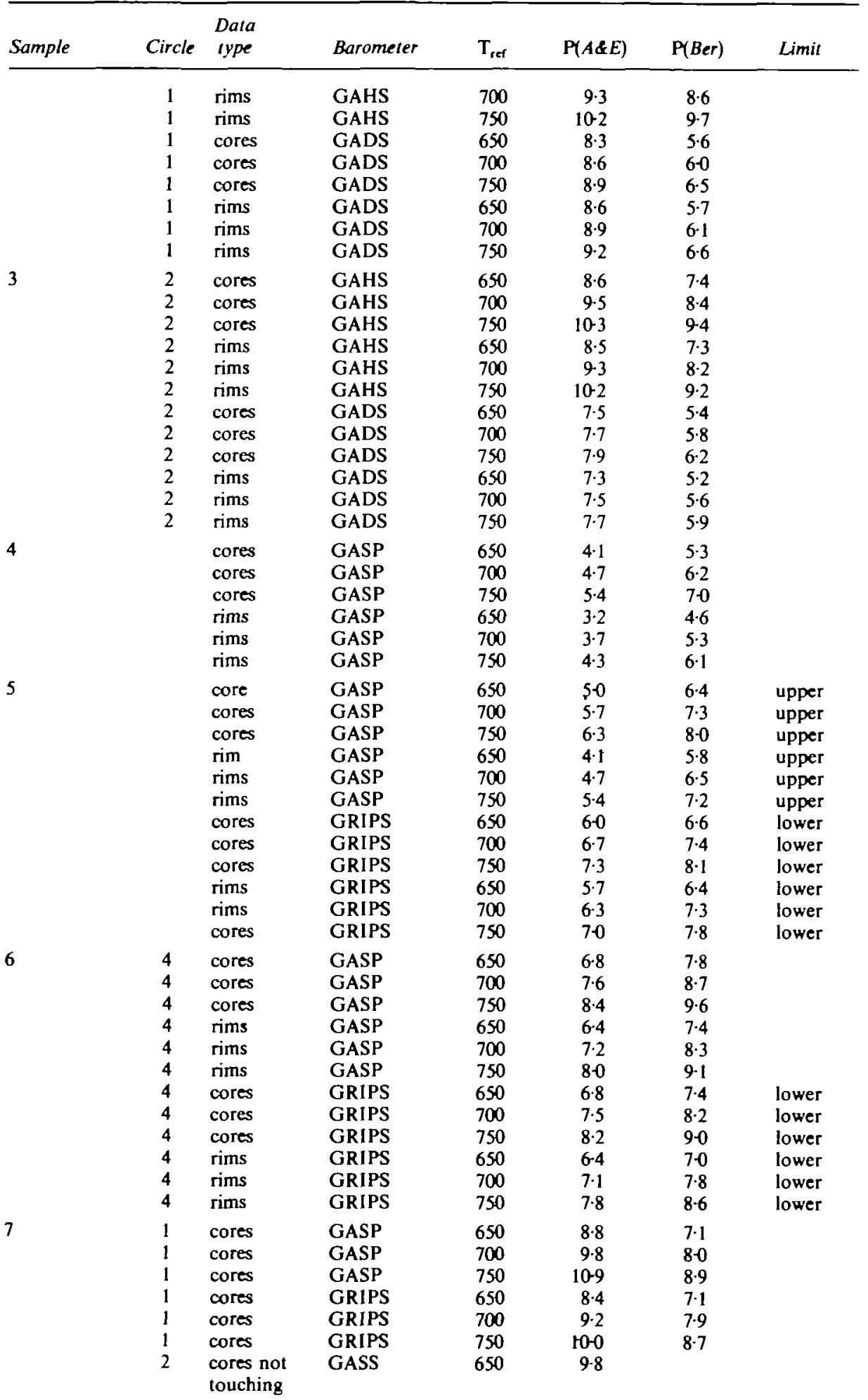


TABLE 9 (Continued)

\begin{tabular}{|c|c|c|c|c|c|c|c|}
\hline Sample & Circle & $\begin{array}{l}\text { Dala } \\
\text { type }\end{array}$ & Barometer & $\mathrm{T}_{\text {rof }}$ & $\mathrm{P}(A \& E)$ & $\mathrm{P}(\mathrm{Ber})$ & Limit \\
\hline & 2 & $\begin{array}{l}\text { cores not } \\
\text { touching }\end{array}$ & GASS & 700 & $11 \cdot 2$ & & \\
\hline & 2 & $\begin{array}{l}\text { cores not } \\
\text { touching }\end{array}$ & GASS & 750 & $12 \cdot 7$ & & \\
\hline & 5 & cores & GASP & 650 & $6 \cdot 9$ & & \\
\hline & 5 & cores & GASP & 700 & $7 \cdot 7$ & & \\
\hline & 5 & cores & GASP & 750 & $8 \cdot 4$ & & \\
\hline & 5 & cores & GRIPS & 650 & 69 & & \\
\hline & 5 & cores & GRIPS & 700 & $7 \cdot 7$ & & \\
\hline & 5 & cores & GRIPS & 750 & $8 \cdot 4$ & & \\
\hline & 5 & $\begin{array}{l}\text { cores } \\
\text { touching }\end{array}$ & GASS & 650 & $10-7$ & & \\
\hline & 5 & $\begin{array}{l}\text { cores } \\
\text { touching }\end{array}$ & GASS & 700 & $12 \cdot 1$ & & \\
\hline & 5 & $\begin{array}{l}\text { cores } \\
\text { touching }\end{array}$ & GASS & 750 & 13.6 & & \\
\hline & 5 & $\begin{array}{l}\text { rims } \\
\text { touching }\end{array}$ & GASS & 650 & $11 \cdot 2$ & & \\
\hline & 5 & $\begin{array}{l}\text { rims } \\
\text { touching }\end{array}$ & GASS & 700 & $12 \cdot 8$ & & \\
\hline & 5 & $\begin{array}{l}\text { rims } \\
\text { touching }\end{array}$ & GASS & 750 & 14.3 & & \\
\hline \multirow[t]{12}{*}{8} & & $\begin{array}{l}\text { high } X_{G r} \\
\text { matrix pl }\end{array}$ & GASP & 650 & 9.8 & $9 \cdot 8$ & \\
\hline & & $\begin{array}{l}\text { high } X_{G r} \\
\text { matrix ol }\end{array}$ & GASP & 700 & $10-8$ & $10-8$ & \\
\hline & & $\begin{array}{l}\text { high } X_{\mathrm{Or}} \\
\text { matrix } \mathrm{pl}\end{array}$ & GASP & 750 & $11 \cdot 8$ & 11.8 & \\
\hline & & $\begin{array}{l}\text { low } X_{\mathrm{Or}} \\
\text { matrix pl }\end{array}$ & GASP & 650 & $5 \cdot 5$ & 6.4 & \\
\hline & & $\begin{array}{l}\text { low } X_{\mathrm{Gr}} \\
\text { matrix pl }\end{array}$ & GASP & 700 & $6 \cdot 2$ & $7 \cdot 2$ & \\
\hline & & low $X_{\mathrm{Or}}$ & GASP & 750 & 70 & 80 & \\
\hline & & $\begin{array}{l}\text { high } X_{\mathrm{Gr}} \\
\text { matrix pl }\end{array}$ & GRIPS & 650 & 80 & $7 \cdot 8$ & lower \\
\hline & & $\begin{array}{l}\text { high } X_{\mathrm{Gr}} \\
\text { matrix pl }\end{array}$ & GRIPS & 700 & 8.8 & $8 \cdot 6$ & lower \\
\hline & & $\begin{array}{l}\text { high } X_{\mathrm{Gr}} \\
\text { matrix pl }\end{array}$ & GRIPS & 750 & 9.5 & 9.4 & lower \\
\hline & & $\begin{array}{l}\text { low } X_{\mathrm{Gr}} \\
\text { matrix pl }\end{array}$ & GRIPS & 650 & 5.6 & $6 \cdot 1$ & lower \\
\hline & & $\begin{array}{l}\text { low } X_{\text {or }} \\
\text { matrix pl }\end{array}$ & GRIPS & 700 & $6 \cdot 3$ & 67 & lower \\
\hline & & $\begin{array}{l}\text { low } X_{G r} \\
\text { matrix pl }\end{array}$ & GRIPS & 750 & 70 & 7.4 & lower \\
\hline \multirow[t]{3}{*}{9} & & cores & GRIPS & 650 & 110 & $10-9$ & \\
\hline & & cores & GRIPS & 700 & 11.9 & 11.9 & \\
\hline & & cores & GRIPS & 750 & $12 \cdot 8$ & $13-0$ & \\
\hline \multirow[t]{10}{*}{10} & & cores & GADS & 650 & $8 \cdot 3$ & 60 & \\
\hline & & cores & GADS & 700 & $8 \cdot 6$ & $6 \cdot 4$ & \\
\hline & & cores & GADS & 750 & 8.9 & 6.9 & \\
\hline & & rims & GADS & 650 & $7 \cdot 7$ & $5 \cdot 4$ & \\
\hline & & rims & GADS & 700 & 80 & 5.8 & \\
\hline & & rims & GADS & 750 & $8 \cdot 2$ & 61 & \\
\hline & & cores & GAHS & 650 & 8.9 & 80 & \\
\hline & & cores & GAHS & 700 & 9.8 & 91 & \\
\hline & & cores & GAHS & 750 & 107 & 102 & \\
\hline & & rims & GAHS & 650 & 8.5 & $7 \cdot 6$ & \\
\hline
\end{tabular}


TABLE 9 (Continued)

\begin{tabular}{|c|c|c|c|c|c|c|c|}
\hline Sample & Circle & $\begin{array}{l}\text { Data } \\
\text { type }\end{array}$ & Barometer & $\mathrm{T}_{\text {ref }}$ & $\mathrm{P}(A \& E)$ & $\mathrm{P}(B e r)$ & Limit \\
\hline & & $\begin{array}{l}\text { rims } \\
\text { rims }\end{array}$ & $\begin{array}{l}\text { GAHS } \\
\text { GAHS }\end{array}$ & $\begin{array}{l}700 \\
750\end{array}$ & $\begin{array}{r}9 \cdot 4 \\
10-3\end{array}$ & $\begin{array}{l}8 \cdot 6 \\
9 \cdot 6\end{array}$ & \\
\hline \multirow[t]{12}{*}{11} & & $\begin{array}{l}\text { high } X_{\mathrm{Gr}} \\
\text { matrix pl }\end{array}$ & GASP & 650 & $10-2$ & $10-1$ & \\
\hline & & $\begin{array}{l}\text { high } X_{\mathrm{Gr}} \\
\text { matrix pl }\end{array}$ & GASP & 700 & $11 \cdot 2$ & $11 \cdot 1$ & \\
\hline & & $\begin{array}{l}\text { high } X_{\mathrm{Gr}} \\
\text { matrix ol }\end{array}$ & GASP & 750 & $12 \cdot 2$ & $12 \cdot 2$ & \\
\hline & & $\begin{array}{l}\text { low } X_{\mathrm{Or}} \\
\text { matrix } \mathrm{pl}\end{array}$ & GASP & 650 & 4.7 & 5.9 & \\
\hline & & low $X_{\mathrm{Gr}}$ & GASP & 700 & $5 \cdot 4$ & 6.6 & \\
\hline & & $\begin{array}{l}\text { low } X_{\mathrm{Gr}} \\
\text { matrix pl }\end{array}$ & GASP & 750 & $6 \cdot 1$ & $7 \cdot 4$ & \\
\hline & & $\begin{array}{l}\text { high } X_{\mathrm{Gr}} \\
\text { matrix pl }\end{array}$ & GRIPS & 650 & 8.5 & $8 \cdot 2$ & lower \\
\hline & & $\begin{array}{l}\text { high } X_{\mathrm{Gr}} \\
\text { matrix pl }\end{array}$ & GRIPS & 700 & $9 \cdot 2$ & 90 & lower \\
\hline & & $\begin{array}{l}\text { high } X_{\mathrm{Gr}} \\
\text { matrix pl }\end{array}$ & GRIPS & 750 & 9.8 & 9.8 & lower \\
\hline & & $\begin{array}{l}\text { low } X_{\mathrm{Gr}} \\
\text { matrix pl }\end{array}$ & GRIPS & 650 & 5.6 & 60 & lower \\
\hline & & $\begin{array}{l}\text { low } X_{\mathrm{Gr}} \\
\text { matrix } \mathrm{pl}\end{array}$ & GRIPS & 700 & $6 \cdot 3$ & $6 \cdot 7$ & lower \\
\hline & & $\begin{array}{l}\text { low } X_{\mathrm{Gr}} \\
\text { matrix pl }\end{array}$ & GRIPS & 750 & 6.9 & $7 \cdot 4$ & lower \\
\hline \multirow[t]{12}{*}{12} & 1 & cores & GASP & 650 & 50 & 6.6 & \\
\hline & 1 & cores & GASP & 700 & $5 \cdot 6$ & $7 \cdot 4$ & \\
\hline & 1 & cores & GASP & 750 & 6.5 & $8 \cdot 1$ & \\
\hline & 1 & rims & GASP & 650 & $4 \cdot 3$ & 7.4 & \\
\hline & 1 & rims & GASP & 700 & 50 & $8 \cdot 2$ & \\
\hline & 1 & rims & GASP & 750 & $5 \cdot 7$ & 90 & \\
\hline & 3 & cores & GASP & 650 & 50 & 6.6 & \\
\hline & 3 & cores & GASP & 700 & 5.6 & $7 \cdot 4$ & \\
\hline & 3 & cores & GASP & 750 & 6.5 & $8 \cdot 2$ & \\
\hline & 3 & cores & GRIPS & 650 & $6 \cdot 3$ & 7.0 & \\
\hline & 3 & cores & GRIPS & 700 & 70 & 7.8 & \\
\hline & 3 & cores & GRIPS & 750 & $7 \cdot 7$ & 8.6 & \\
\hline
\end{tabular}

A\&E-Anovitz \& Essene (1987). Ber-Berman (1990).

The coexistence of spinel and garnet in sample 7 suggests that the equilibrium

$$
\begin{gathered}
\text { hercynite + quartz }=\text { almandine + kyanite } \\
3 \mathrm{FeAl}_{2} \mathrm{O}_{4}+5 \mathrm{SiO}_{2}=\mathrm{Fe}_{3} \mathrm{Al}_{2} \mathrm{Si}_{3} \mathrm{O}_{12}+2 \mathrm{Al}_{2} \mathrm{SiO}_{3}
\end{gathered}
$$

was buffered in the rock. To apply this equilibrium, the experimental calibration of Bohlen $e t$ al. (1986) was used. An ideal mixing model was used for the activity of $\mathrm{FeAl}_{2} \mathrm{O}_{4}$ in spinel. When core analyses of garnet and spinel that are not in contact are used, the calculation yields $11.2 \mathrm{~kb}$ at $700^{\circ} \mathrm{C}$. When core analyses of touching grains of spinel and garnet are used, the calculation yields $12.0 \mathrm{~kb}$ at $700^{\circ} \mathrm{C}$. Pairing adjacent rim analyses places the reaction at $12.8 \mathrm{~kb}$ at $700^{\circ} \mathrm{C}$. The scatter in results is partly due to heterogeneity among spinels. Although major chemical differences among spinels do not seem to correspond to association with garnet, there is some indication that the garnet has exchanged with the touching spinel, as shown by the drop in $m g$-number of the garnet where in contact with spinel. 


\section{Metagabbros}

Three metagabbros were used to gain additional estimates of pressure. Cores and rims of touching garnet, clinopyroxene, and plagioclase were analyzed.

Two barometers:

$$
\begin{gathered}
\text { anorthite }+ \text { hedenbergite }=\text { grossular }+ \text { almandine }+ \text { quartz } \\
3 \mathrm{CaAl}_{2} \mathrm{Si}_{2} \mathrm{O}_{8}+3 \mathrm{CaFeSi}_{2} \mathrm{O}_{6}=2 \mathrm{Ca}_{3} \mathrm{Al}_{2} \mathrm{Si}_{3} \mathrm{O}_{12}+\mathrm{Fe}_{3} \mathrm{Al}_{2} \mathrm{Si}_{3} \mathrm{O}_{12}+3 \mathrm{SiO}_{2}
\end{gathered}
$$

and

$$
\begin{gathered}
\text { anorthite }+ \text { diopside }=\text { grossular }+ \text { pyrope }+ \text { quartz } \\
3 \mathrm{CaAl}_{2} \mathrm{Si}_{2} \mathrm{O}_{8}+3 \mathrm{CaMgSi}_{2} \mathrm{O}_{6}=2 \mathrm{Ca}_{3} \mathrm{Al}_{2} \mathrm{Si}_{3} \mathrm{O}_{12}+\mathrm{Mg}_{3} \mathrm{Al}_{2} \mathrm{Si}_{3} \mathrm{O}_{12}+3 \mathrm{SiO}_{2}
\end{gathered}
$$

have been calculated from available experimental and thermodynamic data by Moecher $e t$ al. (1988). The GAHS reaction was recalculated in this study using the free energy of hedenbergite from Moecher \& Chou (1990). The ideal model described by Moecher et al. (1988) was used to calculate diopside and hedenbergite activities in clinopyroxenes because they showed that it gave the same results as the more elaborate nonideal mixing model of Davidson \& Lindsley (1985). Pressures obtained for the two barometers differ by $0-1-1 \cdot 3 \mathrm{~kb}$ at $650^{\circ} \mathrm{C}$ and $1.0-2.5 \mathrm{~kb}$ at $750^{\circ} \mathrm{C}$ (Table 9). Core pressures for sample 3 are $0 \cdot 1-0.2 \mathrm{~kb}$ higher than rim pressures. Sample 10 gives core pressures $0.4-0.5 \mathrm{~kb}$ higher than rim pressures, and sample 2 yields rim pressures that are $0 \cdot 1-0.3 \mathrm{~kb}$ higher than core pressures. Microprobe counting statistics result in a $\pm 0 \cdot 1-\mathrm{kb}$ precision with these barometers. Given the additional uncertainties in the activity models of garnets, pyroxenes, and feldspars, the calculated pressures between cores and rims of samples 2 and 3 are not regarded as distinguishable.

\section{Comparison of activity models}

Pressures were calculated from GASP and GRIPS with the garnet activity model of Berman (1990) for one or two sets of analyses for each sample. Pressures calculated at $700^{\circ} \mathrm{C}$ with the mixing models of Anovitz \& Essene (1987) and Berman (1990) are compared in Figs $6 \mathrm{a}$ and $6 \mathrm{~b}$. Figure $6 \mathrm{a}$ demonstrates that agreement between pressures calculated with the two models is within $\sim 1 \mathrm{~kb}$ for the GRIPS and GAHS barometers. The deviation is somewhat greater for the GASP barometer and is greatest for the GADS barometer. Figure $6 \mathrm{~b}$ compares the two garnet-clinopyroxene barometers calculated with the two mixing models. The GAHS and GADS barometers are in better agreement when the garnet activities are calculated with the model of Anovitz \& Essene (1987), but use of the Berman mixing model does not change the conclusions of this study. However, the Anovitz \& Essene (1987) model may be more appropriate for the samples in this study as it results in better agreement between the garnet-clinopyroxene barometers.

\section{THERMOMETRY}

\section{Garnet-biotite}

Garnet-biotite thermometry has been applied to five metapelites and one metatonalite (Table 10). The calibrations used are from Ferry \& Spear (1978), Hodges \& Spear (1982), and Indares \& Martignole (1985b). Temperatures were calculated at a pressure range reflecting the barometric results for each sample. All iron in biotite and garnet was assumed to be $\mathrm{Fe}^{2+}$. The calculations yield a wide range of temperatures, as was found by other workers 

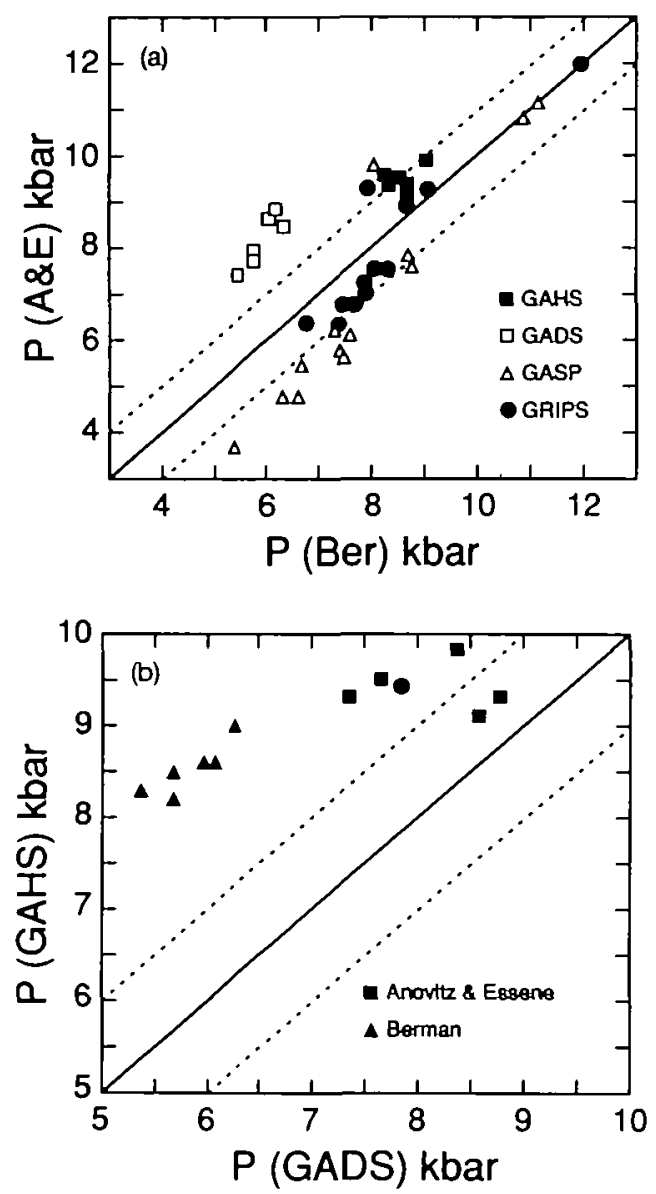

F1G. 6. (a) Comparison of pressures obtaned at $700^{\circ} \mathrm{C}$ with the garnet mixing models of Anovitz \& Essene (1987) and Berman (1990) for at least one pair of analyses from each sample. Perfect agreement between the two models would result in points lying along the diagonal line. Symbols that lie between the two dashed lines indicate agreement within $1 \mathrm{~kb}$. (b) Comparison of pressures obtained at $700^{\circ} \mathrm{C}$ with the GAHS and GADS barometers using the garnet mixing models of Anovitz \& Essene (1987) and Berman (1990).

(e.g., Bohlen \& Essene, 1980; Indares \& Martignole, 1985a; Schreurs, 1985; Chipera \& Perkins, 1988; Edwards \& Essene, 1988; Young et al., 1989). Values obtained with the model of Hodges \& Spear (1982) are similar to those obtained with the model of Ferry \& Spear (1978), whereas the calibration of Indares \& Martignole (1985b) gives lower temperatures. The large spread of temperatures obtained and the late textures of some biotites in metapelites from the Britt domain suggest that garnet-biotite thermometry is not suitable for these rocks.

\section{Garnet-clinopyroxene}

Garnet-clinopyroxene $\mathrm{Fe}-\mathrm{Mg}$ exchange thermometry has been applied to pairs of core and rim analyses from the metagabbros (Table 11). Investigators have proposed various 
TABLE 10

Garnet-biotite temperature $\left(\right.$ in $\left.{ }^{\circ} \mathrm{C}\right)$

\begin{tabular}{|c|c|c|c|c|c|c|}
\hline Sample & Circle & Data type & $F \& S$ & $H \& S$ & $I \& M$ & $\mathbf{P}(k b)$ \\
\hline \multirow[t]{12}{*}{1} & 1 & core $\mathrm{Gt}$-matrix Bi & 730 & 750 & 600 & 6 \\
\hline & & & 730 & 760 & 600 & 7 \\
\hline & & & 730 & 760 & 600 & 8 \\
\hline & 2 & core $\mathrm{Gt}$-matrix $\mathrm{Bi}$ & 710 & 740 & 580 & 6 \\
\hline & & & 710 & 740 & 590 & 7 \\
\hline & & & 720 & 750 & 590 & 8 \\
\hline & 1 & $\operatorname{rim} \mathrm{Gt}-$ near $\mathrm{Bi}$ & 550 & 570 & 440 & 6 \\
\hline & & & 550 & 580 & 444 & 7 \\
\hline & & & 560 & 580 & 450 & 8 \\
\hline & 2 & $\operatorname{rim} \mathrm{Gt}-$ near $\mathrm{Bi}$ & 490 & 520 & 390 & 6 \\
\hline & & & 500 & 520 & 400 & 7 \\
\hline & & & 500 & 520 & 400 & 8 \\
\hline \multirow[t]{4}{*}{7} & 5 & core $\mathrm{Gt}-$ matrix $\mathrm{Bi}$ & 810 & 820 & 720 & 10 \\
\hline & 4 & core $\mathrm{Gt}-$ matrix $\mathrm{Bi}$ & 790 & 790 & 700 & 10 \\
\hline & 1 & core Gt-matrix $\mathrm{Bi}$ & 700 & 700 & 630 & 10 \\
\hline & 4 & rim $\mathrm{Gt}-$ matrix $\mathrm{Bi}$ & 770 & 780 & 690 & 10 \\
\hline \multirow[t]{7}{*}{8} & & high $m g$-number $\mathrm{Gt}$-matrix $\mathrm{Bi}$ & 910 & 950 & 770 & 10 \\
\hline & & & 920 & 960 & 780 & 12 \\
\hline & & & 900 & 930 & 760 & 8 \\
\hline & & medium $m g$-number $\mathrm{Gt}$-matrix $\mathrm{Bi}$ & 710 & 750 & 590 & 7 \\
\hline & & Bi inclusion-touching Gt & 510 & 530 & 390 & 6 \\
\hline & & & 510 & 530 & 390 & 7 \\
\hline & & touching $\mathrm{Gt}-\mathrm{B}_{1}$ & 500 & 530 & 440 & 7 \\
\hline \multirow[t]{4}{*}{11} & & high $m g$-number $\mathrm{Gt}$-matrix $\mathrm{Bi}$ & 760 & 780 & 620 & 6 \\
\hline & & & 780 & 800 & 640 & 11 \\
\hline & & low mg-number $\mathrm{Gt}$-near $\mathrm{Bi}$ & 560 & 580 & 450 & 6 \\
\hline & & & 580 & 600 & 470 & 11 \\
\hline 9 & & core Gt-matrix $\mathrm{Bi}$ & 690 & 810 & 530 & 10 \\
\hline
\end{tabular}

F\&S-Ferry \& Spear (1978). H\&S-Hodges \& Spear (1982). I\&M-Indares \& Martignole (1985b).

versions of garnet-clinopyroxene exchange thermometry, as represented by the reaction

$$
\begin{aligned}
& \text { almandine }+ \text { diopside }=\text { pyrope }+ \text { hedenbergite } \\
& \mathrm{Fe}_{3} \mathrm{Al}_{2} \mathrm{Si}_{3} \mathrm{O}_{12}+3 \mathrm{CaMgSi}_{2} \mathrm{O}_{6}=\mathrm{Mg}_{3} \mathrm{Al}_{2} \mathrm{Si}_{3} \mathrm{O}_{12}+3 \mathrm{CaFeSi}_{2} \mathrm{O}_{6} .
\end{aligned}
$$

Ellis \& Green (1979) considered the effects of pressure, temperature, and grossular component on this reaction. Pattison \& Newton (1989) evaluated the effect of the garnet and clinopyroxene $m g$-number on $K_{\mathrm{D}}$. The experimental calibration of Ellis \& Green (1979) has yielded temperature estimates in reasonable agreement with other thermometers (e.g., Bohlen \& Essene, 1980; Savage \& Sills, 1980; Barnicoat, 1983; Sandiford, 1985; Sanders et al., 1987; Harley, 1988; Anovitz \& Essene, 1990). Application of this thermometer to samples from the southern Britt domain gives temperatures ranging from 620 to $650^{\circ} \mathrm{C}$, somewhat lower than the $700 \pm 50^{\circ} \mathrm{C}$ anticipated for this part of the terrane. However, these results are considerably more reasonable than the temperatures of $440-490^{\circ} \mathrm{C}$ calculated with the equation of Pattison \& Newton (1989), who noted that their thermometer records low temperatures for most high-grade rocks and suggested that this may indicate retrograde resetting. Some resetting of the garnet-clinopyroxene system may have occurred in the Britt samples during their retrograde history. Diffusion data from Chakraborty \& Ganguly (1990) indicate that the $0-1-0.5-\mathrm{cm}$ garnets in the metagabbros should not be able to retain their peak metamorphic compositions at the cooling rates of $2-4^{\circ} \mathrm{C} / \mathrm{Ma}$ indicated for the CGB 
(Cosca, 1989; Cosca et al., 1991). However, resetting of core-core temperatures to $\angle 600^{\circ} \mathrm{C}$ is inconsistent with estimates of the temperatures required for effective garnet diffusion (Woodsworth, 1977; Yardley, 1977; Chakraborty \& Ganguly, 1990). The temperatures that were obtained with the calibration of Pattison \& Newton (1989) may, therefore, reflect systematic errors in their chemical analyses or in extrapolation of the thermometer to temperatures well below the experimental range of $800-1200^{\circ} \mathrm{C}$.

The GAHS and GADS reactions may also be combined to yield reaction (8) (Perkins, 1990). The intersections of GAHS and GADS for the Britt assemblages are shown by the large dots in Fig. 7. Temperatures range from $550^{\circ} \mathrm{C}$ to $\sim 670^{\circ} \mathrm{C}$ and are suggestive of less resetting than implied by application of Pattison \& Newton's calibration. Core to rim changes in temperature are small enough to be within the expected uncertainty in current versions of this thermometer. The calibration of reaction (8) is consistent with GAHS and GADS barometry but is dependent on the solution models chosen. Until a consensus is reached on mixing models for garnets, pyroxenes, feldspars, biotites, and amphiboles, any exchange thermometer involving these phases must be regarded as provisional at best (Essene, 1989).

\section{Staurolite-garnet}

An empirical thermometer based on the Fe-Mg exchange between coexisting staurolite and garnet was calibrated by Fed'kin \& Aranovich (1991). The calibration is based on a comparison with temperatures obtained with the garnet-biotite thermometer from the same samples. The model takes into account the effect of $\mathrm{Zn}$ on the $\mathrm{Fe}-\mathrm{Mg}$ partitioning between staurolite and garnet and corrects for the nonideal character of the $\mathrm{Fe}-\mathrm{Mg}$ interaction. The thermometer was only calibrated between $\sim 450^{\circ} \mathrm{C}$ and $600^{\circ} \mathrm{C}$ and therefore has to be extrapolated to be applicable in pelites from the southern Britt domain. Coexisting garnet and staurolite from sample 7 yield temperatures of $650^{\circ} \mathrm{C}$.

\section{Staurolite-hercynite}

The coexistence of staurolite, quartz, garnet, and kyanite suggests that the reaction

$$
\begin{gathered}
\text { staurolite }+ \text { quartz }=\text { almandine }+ \text { kyanite }+\mathrm{H}_{2} \mathrm{O} \\
12 \mathrm{H}_{2} \mathrm{Fe}_{2} \mathrm{Al}_{9} \mathrm{Si}_{3.75} \mathrm{O}_{24}+25 \mathrm{SiO}_{2}=8 \mathrm{Fe}_{3} \mathrm{Al}_{2} \mathrm{Si}_{3} \mathrm{O}_{12}+46 \mathrm{Al}_{2} \mathrm{SiO}_{5}+12 \mathrm{H}_{2} \mathrm{O}
\end{gathered}
$$

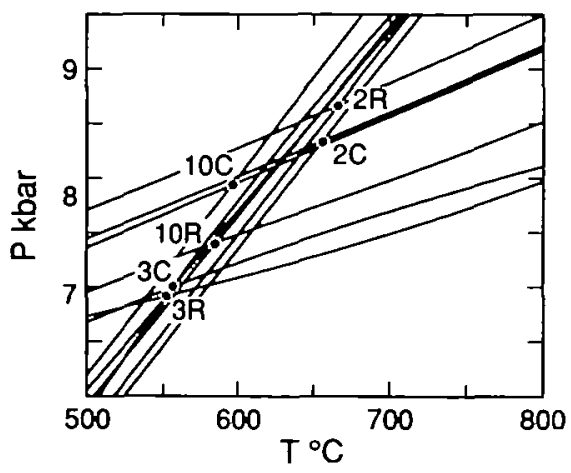

FIG. 7. Garnet-clinopyroxene-plagioclase-quartz barometry for core and rim analyses of samples 2, 3, and 10 using the GADS and GAHS barometers. Steeply sloping lines represent GAHS pressures; lines with a shallow slope represent GADS pressures. Dots mark the intersection of the two barometers for core and rim analyses for each

sample and represent temperature estimates from the garnet-clinopyroxene $\mathrm{Fe}-\mathrm{Mg}$ exchange reaction. 
may be buffered in sample 7. Textures of spinel overgrowing staurolite (Fig. 3c) also suggest the prograde breakdown of staurolite by the following reaction:

$$
\begin{gathered}
\text { staurolite }=\text { hercynite }+ \text { kyanite }+ \text { quartz }+\mathrm{H}_{2} \mathrm{O} \\
4 \mathrm{H}_{2} \mathrm{Fe}_{2} \mathrm{Al}_{9} \mathrm{Si}_{3 \cdot 75} \mathrm{O}_{24}=8 \mathrm{FeAl}_{2} \mathrm{O}_{4}+10 \mathrm{Al}_{2} \mathrm{SiO}_{5}+5 \mathrm{SiO}_{2}+4 \mathrm{H}_{2} \mathrm{O} .
\end{gathered}
$$

These two reactions were modeled with available experimental (Richardson, 1968; Ganguly, 1972; Rao \& Johannes, 1979; Dutrow \& Holdaway, 1989) and thermodynamic (Gibbons et al., 1981; Hemingway \& Robie, 1984) data (cf. Tuccillo, 1990). These reactions are very sensitive to solid solutions and to variations in $a_{\mathrm{H}_{2} \mathrm{O}}$; at present, they cannot be regarded as dependable for thermometry. Because of difficulties in obtaining estimates for $a_{\mathrm{H}_{2} \mathrm{O}}$, the positions of reactions (9) and (10) have been calculated for $a_{\mathbf{H}_{2} \mathrm{O}}$ values of $0 \cdot 2,0.4$, and 0.6 in Fig. 8, corrected for the effects of staurolite, garnet, and hercynite solid solution, for two sets of analyses. Tuccillo (1990) concluded that correcting reaction (10) for solid solution places the reaction at temperatures that are probably too low. Temperature information gained from reactions $(9)$ and $(10)$ is therefore considered approximate. However, the $P-T$ path followed by the staurolite-bearing metapelite is inferred to have crossed the univariant curve in a prograde sense, i.e., similar to the path shown in Fig. 9.

\section{GEOCHRONOLOGY}

Information on the timing of metamorphism and deformation was obtained from minerals with different closure temperatures, using $\mathrm{U}-\mathrm{Pb}$ isotope systematics. To assure the metamorphic origin of monazite, allanite, and garnet, these minerals were separated from metapelites only. As indicated by the preservation of prograde zoning in garnets from these

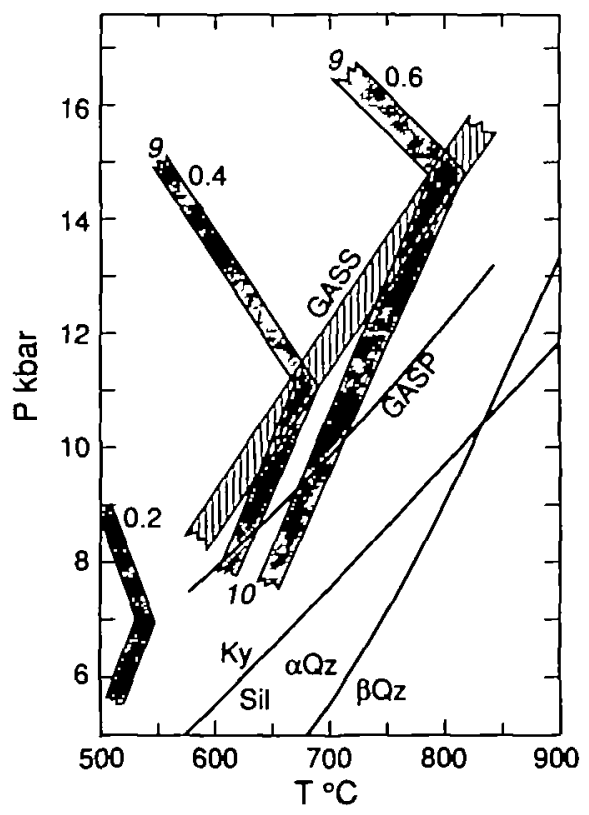

FIG. 8. Upper limits of staurolite stability for sample 7 as calculated from reactions (9) and (10) at $a_{\mathrm{HrO}}$ values of 02 , $0-4$, and 0-6 for two sets of analyses. Also shown are GASS values obtained from the two sets of analyses and maximum values obtained from GASP barometry for this sample. 
TABLE 11

Garnet-clinopyroxene temperature $\left(\right.$ in $\left.^{\circ} \mathrm{C}\right)$

\begin{tabular}{llcc}
\hline Sample & Type & $E \& G$ & $P \& N$ \\
\hline 10 & cores & 620 & 470 \\
& rims & 620 & 480 \\
2 & cores & 620 & 470 \\
& rims & 630 & 490 \\
3 & cores & 650 & 480 \\
& rims & 620 & 440 \\
\hline
\end{tabular}

E\&G-Ellis \& Green (1979). P\&N-

Pattison \& Newton (1989).

metapelites, it is most likely that during metamorphism the peak temperature did not exceed $750^{\circ} \mathrm{C}$, otherwise the prograde Ca zoning in these $\sim 0.5-\mathrm{cm}$ garnets would have been erased (Chakraborty \& Ganguly, 1990). Under these conditions it is expected that the garnets and possibly the allanite record the time of prograde metamorphism (Mezger et al., 1989). As the closure temperature for monazite is $\sim 725^{\circ} \mathrm{C}$ (Parrish, 1991), this mineral may record either prograde metamorphism or an early cooling age.

The core of the allanite from the southern Britt domain yielded a concordant age of $1450 \pm 1 \mathrm{Ma}$, and a fragment from within $2 \mathrm{~mm}$ of the rim yielded a ${ }^{207} \mathrm{~Pb} /{ }^{206} \mathrm{~Pb}$ age of $1435 \pm 2 \mathrm{Ma}$ and is $\sim 0.5 \%$ discordant (Table 12 ). Although powder X-ray diffraction indicates that the allanite is completely metamict, its core seems to be unaffected by $\mathrm{U}$ or $\mathrm{Pb}$ loss. The age of $1450 \mathrm{Ma}$ for the core is taken as the time of growth of this large single allanite crystal. This age is corroborated by igneous zircons from a hornblendé-garnet-biotite granitoid orthogneiss in the Britt domain that yielded a $\mathrm{U}-\mathrm{Pb}$ age of $1450 \mathrm{Ma}$ for the intrusion of the protolith (van Breemen et al., 1986). The agreement of the U-Pb age from a pluton and that from a metamorphic allanite indicates that magmatism may have played an important role in the heat budget during the early metamorphism.

Garnet from sample 8 gave a $\mathrm{K}$-feldspar-garnet $\mathrm{Pb}-\mathrm{Pb}$ age of $1396 \pm 6 \mathrm{Ma}$, and monazite from the same sample yielded an age of $\sim 1060 \mathrm{Ma}$. The monazite age is identical to the minimum age obtained from sphene from the Britt domain (Table 12). Monazite occurs as small $(\sim 10 \mu \mathrm{m})$ inclusions in the major rock-forming minerals including garnet, and is therefore considered to be contemporaneous with these minerals. In addition, the larger $(\sim 100 \mu \mathrm{m})$ monazites, which were used for dating, occur in the matrix of the metapelite. The significantly younger age for the monazites and the sphene compared with the garnet indicates that these monazites record a cooling age or were reset during a younger metamorphic episode. However, cooling over $\sim 300-400 \mathrm{Ma}$ is considered unlikely, and it is more realistic that the southern Britt domain underwent two distinct metamorphic episodes. The first episode occurred at $\sim 1450 \mathrm{Ma}$ and a second one at $\sim 1060 \mathrm{Ma}$. The second metamorphic episode is apparently recorded in garnets from within the Parry Sound Shear Zone (Fig. 2). A garnet sample extracted from a metapelite (sample 4) that was sheared under at least amphibolite facies conditions yielded an age of $1123 \pm 3 \mathrm{Ma}$. This age is consistent with zircon ages of 1159-1121 Ma from syn-deformational pegmatites within the shear zone (van Breemen et al, 1986). A sphene from a quartz-sphene vein from within the same shear zone yielded a minimum age of $1078 \mathrm{Ma}$. This age and the discordance of this sphene may indicate that the fault was active well past the time recorded by zircons and garnet. Monazite from the Parry Sound domain within the vicinity of the shear zone yielded an age of 1157 


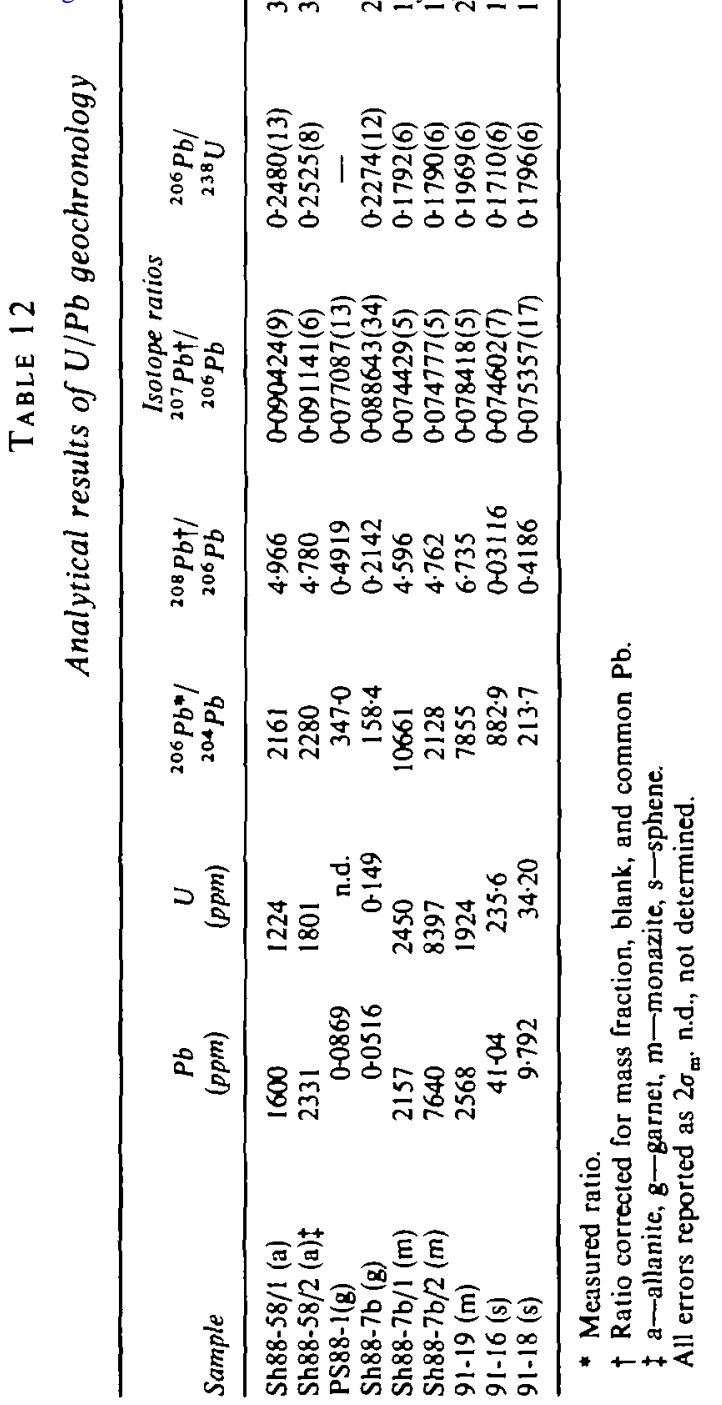


$\pm 1 \mathrm{Ma}$. This age is not recorded in minerals from the Britt domain and indicates that the Parry Sound domain experienced its last high-grade metamorphism before the last metamorphic episode that affected the Britt domain.

The ${ }^{40} \mathrm{Ar} /{ }^{39} \mathrm{Ar}$ data of Culshaw et al. (1991) appear to be related to slow cooling from the second event. These workers reported ${ }^{40} \mathrm{Ar} /{ }^{39} \mathrm{Ar}$ plateau ages of $964 \pm 5 \mathrm{Ma}$ and $969 \pm 5 \mathrm{Ma}$ for hornblendes from the southern Britt domain, located $5-10 \mathrm{~km}$ west of our samples. In addition, a muscovite ${ }^{40} \mathrm{Ar} /{ }^{39} \mathrm{Ar}$ plateau age of $904 \pm 3 \mathrm{Ma}$ was also reported in this area by Culshaw et al. (1991). If the second metamorphic event attained $700 \pm 50^{\circ} \mathrm{C}$ in the southern Britt domain, combination of the available thermochronological data with reasonable blocking temperatures for hornblende $\left(480 \pm 40^{\circ} \mathrm{C}\right)$ and muscovite $\left(350 \pm 50^{\circ} \mathrm{C}\right)$ yields cooling rates of $2-5^{\circ} \mathrm{C} / \mathrm{Ma}$. These time-averaged rates are slow but within the range inferred by Cosca et al. (1991) elsewhere in the Central Gneiss Belt.

\section{DISCUSSION}

Calculated temperatures, pressures, mineral ages, and textural information help to constrain the $P-T-t$ path for the southern Britt domain. The complexly zoned garnets in samples 8 and 11 provide valuable information. The $\mathrm{Ca}$ zoning has been interpreted to represent late growth or early retrograde zoning under conditions of decreasing pressure, whereas the core to rim variations in $m g$-number and $\mathrm{Mn}$ are interpreted to be a superimposed diffusional effect during retrogression (cf. Tuccillo et al., 1990). The 5-6 kb pressure difference recorded by the $\mathrm{Ca}$ zoning is consistent with textural evidence of sillimanite overgrowing kyanite (Fig. 9). It is likely that the complex garnets in samples 8 and 11 grew during the first metamorphism at $\sim 1450 \mathrm{Ma}$ and acquired their zoning in major element composition by incomplete re-equilibration during the second regional metamorphism at $\sim 1060 \mathrm{Ma}$, as obtained from $\mathrm{U} / \mathrm{Pb}$ data on monazite and sphene. This second metamorphism reached temperatures that were sufficient to reset the $\mathrm{U}-\mathrm{Pb}$ age of

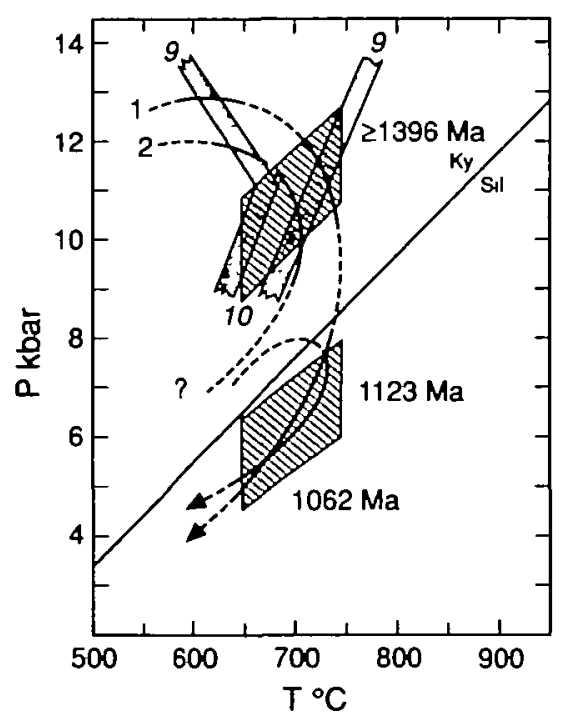

FIG. 9. Hypothetical $P-T$ paths for the Britt domain. Striped parallelograms represent pressure constraints from garnet zoning. Shaded reguons represent constraints from staurolite breakdown equilibria. 
the monazite, but they were not high enough nor persisted long enough to rehomogenize completely the major elements in garnets from metapelites. This observation is consistent with peak metamorphic conditions of $\sim 700^{\circ} \mathrm{C}$ for the second metamorphism. The garnet age of $1396 \mathrm{Ma}$ is significantly younger than the allanite age of $1450 \mathrm{Ma}$. This difference may be due to partial resetting of the $\mathrm{U}-\mathrm{Pb}$ systematics in garnet or may be the result of additional garnet growth during the second metamorphism. As it is currently not possible to separate different parts of the garnets, whole garnets were used for analysis, and it is not possible to distinguish among the possibilities. However, the zoning pattern exhibited by the garnets (Tuccillo et al., 1990; fig. 1) suggests that the larger garnets may have formed by the coalescence of smaller grains. It is therefore considered most likely that some garnet growth that was probably associated with grain coalescence occurred during the second episode.

Barometry from other garnet-bearing samples presented in this paper is consistent with the results from the zoned garnets. Pressures range from somewhat higher $\left(12.9 \mathrm{~kb}\right.$ at $\left.700^{\circ} \mathrm{C}\right)$ than the maximum pressures obtained from samples 8 and 11 to somewhat lower $(4.7 \mathrm{~kb}$ at $700^{\circ} \mathrm{C}$ ) than the minimum values from the two samples. This may indicate that garnets in various samples have equilibrated at different pressures along the $P-T$ path, that partial resetting has occurred during the second metamorphism, or that $P-T$ paths varied across the southern Britt domain.

In addition to garnet barometry, part of the $P-T$ path of the southern Britt domain is considered by staurolite equilibria. Textural relationships document the breakdown of staurolite in sample 7 [reactions (9) and (10)]. Because staurolite is a hydrous mineral, its breakdown involves dehydration and will proceed under conditions of increasing temperature. Figure 9 summarizes the limits on the prograde path from reactions (9) and (10) as well as GASS and GASP barometry for sample 7 (Fig. 8).

The tectonic significance of any $P-T$ path is constrained by information on time. As noted above, a time interval of $\sim 300-400 \mathrm{Ma}$ between ages of several minerals, including garnets with distinct textural characteristics, suggests that the Britt domain was probably affected by at least two thermal events. Figure 9 illustrates two possible $P-T$ trajectories that are consistent with all the data. Both pass through the region defined by reactions (9) and (10) in the kyanite field for $a_{\mathrm{H}_{2} \mathrm{O}}$ of $0-2-0 \cdot 6$, as well as the high- and low-pressure conditions calculated from garnet zoning, and both cross from the kyanite field into the sillimanite field. Path 1 is a simple way to join the $P-T$ data but is unlikely to represent a realistic path because of the need for nearly isothermal conditions that lasted $\sim 300 \mathrm{Ma}$. An alternative path (2) suggests the occurrence of two distinct metamorphic episodes for the Britt domain. One possible interpretation of the $P-T$ data and the mineral ages is that the Parry Sound domain experienced high-grade metamorphism at $\sim 1160 \mathrm{Ma}$, which coincides with the beginning of thrusting of the Parry Sound over the Britt domain along the Parry Sound Shear Zone. Thrusting lasted past $1078 \mathrm{Ma}$, as indicated by the sphene age from the shear zone, and it led to the second metamorphism in the Britt domain, which culminated at $\sim 1060 \mathrm{Ma}$. Thrusting may have been episodic and may have been contemporaneous with thrusting in the Central Metasedimentary Belt Boundary Zone to the south (van Breemen et al., 1986; fig. 1). Although path 2 is not yet completely documented, it emphasizes the complex and protracted history for the Britt domain.

Combining the mineral ages with the petrographic observations leads to the suggestion that the rocks from the southern Britt domain experienced upper amphibolite facies conditions during each of these thermal episodes. The earlier metamorphism at $\sim 1450 \mathrm{Ma}$ reached peak conditions of at least $10 \mathrm{~kb}$ (Tuccillo et al., 1990) and is consistent with the occurrence of early kyanite. The second metamorphism, which culminated at $\sim 1060 \mathrm{Ma}$, reset the $\mathrm{U}$-Pb systematics of monazite and sphene but did not completely re-equilibrate the 
garnets. Thus, the temperatures reached during this second metamorphism could not have exceeded $\sim 700^{\circ} \mathrm{C}$. As indicated by geobarometry and the overgrowths of sillimanite on kyanite, the rocks equilibrated within the stability field of sillimanite during the second episode. Interpretations based on a single metamorphic event at $\sim 1.1 \mathrm{Ga}$ (e.g., Tuccillo et al., 1990) are regarded as over-simplified. Future interpretations of this area will have to consider at least two events rather than a single event at $\sim 1 \cdot 1 \mathrm{Ga}$. Anovitz \& Essene (1990) presented a $P-T$ path for the Parry Sound domain that has a shallower slope but is otherwise similar to path 1. Anovitz (1991) used Al zoning in pyroxenes and feldspars to obtain a decompressive retrograde path for the Parry Sound domain, and modeling by Anovitz \& Chase (1990) suggested that this decompressive path is associated with extension. This interpretation, however, is questionable for the southern Britt domain in light of geochronologic data presented in this paper.

\section{ACK NOWLEDGEMENTS}

This study was supported by National Science Foundation Grants EAR-82-12764, EAR88-05083, EAR-89-03805, and EAR-90-04302, Sigma Xi, and the Scott Turner Fund at the University of Michigan. We thank A. Davidson, N. G. Culshaw, and P. Wallace for valuable field guidance and support, L. M. Anovitz for helpful discussions, C. Henderson for scanning electron microscope and microprobe training, and J. Elliot for field assistance. N. G. Culshaw kindly provided samples 3 and 12. J. Hinchcliff made excellent thin sections and S. L. Busch provided computer assistance. Reviews by J. L. Hayob, D. G. Palais, J. Selverstone, and E. D. Young improved the paper significantly. This paper is Contribution 482 from the Mineralogical Laboratory, Department of Geological Sciences, University of Michigan.

\section{REFERENCES}

Anderson, D. E., \& Olimpio, J. C., 1977. Progressive homogenization of metamorphic garnets, South Morar, Scotland: evidence for volume diffusion. Can. Miner. 15, 205-16.

Anovitz, L. M., 1991. Al-zoning in pyroxene and plagioclase: window on late to prograde to early retrograde $P-T$ paths in granulite terranes. Am. Miner. 76, 1328-43.

- Chase, C. G., 1990. Implications of postthrusting extension and underplating for $P-T-t$ paths in granulite terranes-a Grenville example. Geology 18, 466-9.

_- Essene, E. J., 1987. Compatibility of geobarometers in the system $\mathrm{CaO}-\mathrm{FeO}-\mathrm{Al}_{2} \mathrm{O}_{3}-\mathrm{SiO}_{2}-\mathrm{TiO}_{2}$ (CFAST): implications for garnet mixing models. J. Geol. 95, 633-45.

-1990 . Thermobarometry and pressure-temperature paths in the Grenville Province of Ontario. $J$. Petrology 31, 197-241.

Barnicoat, A. C., 1983. Metamorphism of the Scourian Complex, N. W. Scotland. J. Metamorphic Geol. 1, $163-82$. Berman, R. G. 1990. Mixing properties of $\mathrm{Ca}-\mathrm{Mg}-\mathrm{Fe}-\mathrm{Mn}$ garnets. Am. Miner. 75, 328-433.

Blackbum, W. H., 1969. Zoned and unzoned garnets from the Grenville gneisses around Gananoque, Ontario. Can. Miner. 9, 691-8.

Bohlen, S. R., 1990. The value and limitations of thermobarometry: a partisan view. Geol. Soc. Am. Abstr. Prog. 22, 71 .

- Dollase, W. A., \& Wall, V. J., 1986. Calibration and applications of spinel equilibria in the system $\mathrm{FeO}-\mathrm{Al}_{2} \mathrm{O}_{3}-\mathrm{SiO}_{2}$. J. Petrology 27, 1143-56.

- Essene, E. J., 1977. Feldspar and oxide thermometry of granulites in the Adirondack Highlands. Contr. Miner. Petrol. 62, 153-69.

-1980. Evaluation of coexisting gamet-biotite, garnet-clinopyroxene and other $\mathrm{Mg}-\mathrm{Fe}$ exchange thermometers in Adirondack granulites. Geol. Soc. Am. Bull. 91, 685-719.

- Liotta, J. J., 1986. A barometer for garnet amphibolites and garnet granulites. J. Petrology 27, $1025-56$.

_- Valley, J. W., \& Essene, E. J., 1985. Metamorphism in the Adirondacks. I. Petrology, pressure, and temperature. Ibid. 26, 971-92.

Brown, M. \& Earle, M. M., 1983. Cordierite-bearing schists and gneisses from Timor, eastern Indonesia: $P-T$ conditions of metamorphism and tectonic implications. J. Metamorphic Geol. 1, 183-203. 
Chakraborty, S., \& Ganguly, J., 1990. Compositional zoning and cation diffusion in garnets. In: Ganguly, J. (ed.) Diffusion, Atomic Ordering and Mass Transport: Selected Topics in Geochemistry. Adoances in Physical Geochemistry, 8. New York: Springer-Verlag, 120-75.

Chamberlain, C. P., \& Lyons, J. B., 1983. Pressure, temperature and metamorphic zonation studies of pelitic schists in the Merrimack synclinorium, south-central New Hampshire. Am. Miner. 68, 530-40.

Chupera, S. J., \& Perkins, D, 1988. Evaluation of biotste-garnet geothermometers-application to the English River Subprovince, Ontario. Contr. Miner. Petrol. 98, 40-8.

Cosca, M. A., 1989. Cooling and inferred uplift/erosion history of the Grenville orogen, Ontario: constraints from ${ }^{40} \mathrm{Ar} /{ }^{39} \mathrm{Ar}$ thermochronology. Ph.D. Thesis, University of Michigan.

- Sutter, J. F, \& Essene, E. J., 1991. Cooling and inferred uplift/erosion history of the Grenville Orogen, Ontario: constraints from ${ }^{40} \mathrm{Ar} /{ }^{39} \mathrm{Ar}$ thermochronology. Tectonics 10, 959-77.

Culshaw, N. G., Corrigan, D., Drage, J., \& Wallace, P., 1988. Georgian Bay geological synthesis: Key Harbour to Dillon, Grenville Province of Ontario. Geol. Surv. Can. Spec. Paper 88-1C, 129-33.

Culshaw, N. G., Reynolds, P. H., \& Check, G., 1991. A ${ }^{40} \mathrm{Ar} /{ }^{39} \mathrm{Ar}$ study of post-tectonic cooling in the Britt domain of the Grenville Province, Ontario. Earth Planet. Sci. Lett. 105, 405-15.

Davidson, A., 1984. Tectonic boundaries within the Grenville Province of the Canadian Shield. J. Geodyn. 1, 433-44.

1986. Grenville Front relationships near Killarney, Ontario. In: Moore, J. M., Davidson, A., \& Baer, A. J. (eds.) The Grenville Province. Geol. Assoc. Can. Spec. Paper 31, 107-17.

-Culshaw, N. G., \& Nadeau, L, 1982. A tectono-metamorphic framework for part of the Grenville Province, Parry Sound region, Ontario. In: Current Research, Part A. Geol. Surv. Can. Spec. Paper 82-1A, 175-90.

Davidson, P. M., \& Lindsley, D. H., 1985. Thermodynamic analysis of quadrilateral pyroxenes. Part II: Model calibration from experiments and application to geothermometry. Contr. Miner. Petrol. 91, 390-404.

Dutrow, B. L., \& Holdaway, M. J., 1989. Experimental determination of the upper thermal stability of Fe-staurolite + quartz and medium pressures. J. Petrology 30, 229-48.

Easton, R. M., 1986. Geochronology of the Grenville Province. Part I: Compilation of data; Part II: Synthesis and interpretation. In: Moore, J. M., Davidson, A., \& Baer, A. J. (eds.) The Grenville Province. Geol. Assoc. Can. Spec. Paper 31, 191-207.

Edwards, $\mathbf{R}$ L., \& Essene, E. J., 1988. Pressure, temperature and C-O-H fluid fugacities across the amphibolite-granulite transition, northwest Adirondack Mountains, New York. J. Petrology 29, 39-72.

Ellis, D. J., \& Green, D. H., 1979. An experimental study of the effect of Ca upon garnet-clinopyroxene Fe-Mg exchange equilibria. Contr. Miner. Petrol. 71, 13-22.

England, P. C., \& Thompson, A. B., 1984. Pressure-temperature-time paths of regional metamorphism I. Heat transfer during the evolution of regions of thickened continental crust. J. Petrology 25, 894-928.

Essene, E. J., 1989. The current status of thermobarometry in metamorphic rocks. In: Daly, J. S., Cliff, R. A., \& Yardley, B. W. D. (eds.) Evolution of Metamorphic Belts. Geol. Soc. Lond. Spec. Publ. 43, 1-44.

Fed'kin, V. V, \& Aranovich, L. Ya., 1991. A new thermodynamic model for the staurolite-garnet geothermometer. Geochem. Int. x, 146-50.

Ferry, J. M., \& Spear, F. S., 1978. Experimental calibration of the partitioning of Fe and $\mathrm{Mg}$ between biotite and garnet. Contr. Miner. Petrol. 66, 113-17.

Frost, B. R., \& Chacko, T., 1989. The granulite uncertainty principle: limitations on thermobarometry in granulites. J. Geol. $97,435-50$.

Fuhrman, M. L., \& Lindsley, D. H., 1988. Ternary-feldspar modeling and thermometry. Am. Miner. 73, $201-15$.

Ganguly, J., 1972. Staurolite stability and related parageneses: theory, experiments, and applications. J. Petrology 13, 335-65.

- Saxena, S. K., 1984. Mixing properties of aluminosilicate garnets: constraints from natural and experimental data and applications to geothermobarometry. Am. Miner. 69, 88-97.

Ghent, E. D., Knitter, C. C., Raeside, R. P., \& Stout, M. Z., 1982. Geothermometry and geobarometry of pelitic rocks, upper kyanite and sillimanite zones, Mica Creek area, British Columbia. Can. Miner. 20, 295-305.

Gibbons, K., Dempsey, M. J, \& Henderson, C. M. B, 1981. The thermal expansion of staurolite, $\mathrm{Fe}_{4} \mathrm{Al}_{18} \mathrm{Si}_{8} \mathrm{O}_{44}(\mathrm{OH})_{4}$. Miner. Mag. 44, 69-72.

Grant, J. A., \& Weiblen, P. W, 1971. Retrograde zoning in garnet near the second sillimanite isograd. Am. J. Sci. 270, 281-96.

Harley, S. L., 1988. Proterozoic granulites from the Rauer Group, East Antarctica. I. Decompressional pressure-temperature paths deduced from mafic and felsic gneisses. J. Petrology 29, 1059-95.

Hemingway, B. S $\neg$ Robie, R. A., 1984. Heat capacity and thermodynamic functions for gehlenite and staurolite: with comments on the Schottky anomaly in the heat capacity of staurolite. Am. Miner. 69, 307-18.

Hodges, K. V. \& Royden, R. L., 1984. Geologic thermobarometry of retrograded metamorphic rocks: an indication of the uplift trajectory of a portion of the northern Norwegian Caledonides. J. Geophys. Res. 89, 7077-90.

- Spear, F. S., 1982. Geothermometry, geobarometry and the $\mathrm{Al}_{2} \mathrm{SiO}_{3}$ triple point at Mt. Moosilauke, New Hampshire. Am. Miner. 67, 1118-34.

Holdaway, M. J., Dutrow, B. L., \& Shore, P., 1986. A model for the crystal chemistry of staurolite. Ibid. 71, $1142-59$.

Hollister, L. S., 1966. Garnet zoning: an interpretation based on the Rayleigh fractionation model. Science 154, $1647-51$. 
Indares, A., \& Martignole, J., 1985a. Biotite-garnet geothermometry in granulite-facies rocks-evaluation of equilibrium criteria. Can. Miner. 23, 187-93.

1985b. Biotite-garnet geothermometry in the granulite facies: the influence of Ti and Al in biotite. $\mathrm{Am}$. Miner. 70, 272-8.

Koziol, A. M., \& Newton, R. C., 1988. Redetermination of the anorthite breakdown reaction and improvement of the plagioclase-garnet-Al $\mathrm{SiO}_{5}$-quartz geobarometer. Ibid. 73, 216-23.

Mezger, K., Bohlen, S. R., \& Hanson, G. N., 1990 . Metamorphic history of the Archean Pikwitonei Granulite Domain and the Cross Lake Subprovince, Superior Province, Manitoba, Canada. J. Petrology 31, 483-517.

- Hanson, G. N., \& Bohlen, S. R., 1989. U-Pb systematics of garnet: dating the growth of garnet in the late Archean Pikwitonei granulite domain at Cauchon and Natawahunan Lakes, Manitoba, Canada. Contr. Miner. Petrol. 101, 136-48.

Moecher, D. P., \& Chou, I-M., 1990. Experimental investigation of andradite and hedenbergite equilibria employing the hydrogen sensor technique, with revised estimates of $\Delta G_{298}$ for andradite and hedenbergite. $A m$. Miner. 75, 1327-41.

- Essene, E. J., \& Anovitz, L. M., 1988. Calculation and application of clinopyroxene-gametplagioclase-quartz geobarometers. Contr. Miner. Petrol. 100, 92-106.

_- Perkins, D. III, Leier-Englehardt, P. J., \& Medaris, L. G., Jr., 1986. Metamorphic conditions of late Archean high-grade gneisses, Minnesota River Valley, U.S.A. Can. J. Earth Sci. 23, 633-45.

Parrish, R. R., 1991. U-Pb dating of monazite and its application to geological problems. Ibid. 27, 1431-50.

Pattison, D. R. M, \& Newton, R. C., 1989. Reversed experimental calibration of the garnet-clinopyroxene Fe-Mg exchange thermometer. Contr. Miner. Petrol. 101, 87-103.

Perchuk, L., Nozhkin, A., \& Gerya, T., 1989. Petrology and retrograde $P-T$ path in granulites of the Kanskaya Formation, Yenisey Range, eastern Siberia. J. Metamorphic Geol. 7, 599-617.

Perkins, D., 1990. Thermometry and barometry of mafic granulites based on garnet-clinopyroxeneplagioclase-quartz assemblages. In: Vielzeuf, D, \& Vidal, Ph. (eds.) Granulites and Crustal Evolution. NATO Advanced Sclences Institutes, Series C: Mathematical and Physical Sciences, 311. Dordrecht: Kluwer, 435-49.

Rao, B. B., \& Johannes, W., 1979. Further data on the stability of staurolite + quartz and related assemblages. Neues Jahrb. Miner. Monatsh. 10, 437-47.

Richardson, S. W., 1968. Staurolite stability in a part of the system Fe-Al-Si-O-H. J. Petrology 9, 467-88.

Sanders, I. S., Daly, J. S., \& Davies, G. R., 1987. Late Proterozoic high-pressure granulite facies metamorphism in the north-east Ox inlier, north-west Ireland. J. Metamorphic Geol. 5, 69-85.

Sandiford, M, 1985. The metamorphic conditions of granulites at Fyfe Hills: implications for Archean crustal thickness in Enderby Land, Antarctica. Ibid. 3, 155-78.

Savage, D., \& Sills, J. D., 1980. High pressure metamorphism in the Scourian of NW Scotland: evidence from garnet granulites. Contr. Miner. Petrol. 74, 153-63.

Schreurs, J., 1985. Prograde metamorphism of metapelites, garnet-biotite thermometry and prograde changes of biotite chemistry in high-grade rocks of West Uusimaa, southwest Finland. Lithos 18, 69-80.

Schwerdtner, W. M., 1987. Interplay between folding and ductile shearing in the Proterozoic crust of the Muskoka-Parry Sound region, central Ontario. Can. J. Earth Sci. 24, 1507-25.

Selverstone, J., \& Chamberlain, C. P., 1990. Apparent isobaric cooling paths from granulites: two counterexamples from British Columbia and New Hampshire. Geology 18, 307-10.

- Spear, F. S., 1985. Metamorphic $P-T$ paths from pelitic schists and greenstones from the south-west Tauern Window, Eastern Alps. J. Metamorphic Geol. 3, 439-65.

- Franz, G., \& Morteani, G, 1984. High-pressure metamorphism in the SW Tauern Window, Austria: $P-T$ paths from homblende-kyanite-staurolite schists. J. Petrology 25, 501-31.

Spear, F. S., 1989. Relative thermobarometry and metamorphic $P-T$ paths. In: Daly, J. S., Cliff, R. A., \& Yardley, B. W. D. (eds.) Evolution of Metamorphic Belts. Geol. Soc. Lond. Spec. Publ. 43, 63-81.

-.-Selverstone, J., 1983. Quantitative $P-T$ paths from zoned minerals: theory and tectonic applications. Contr. Miner. Petrol. 83, 348-57.

Hickmott, D., Crowley, P., \& Hodges, K. V., 1984. P-T paths from garnet zoning a new technique for deciphering tectonic processes in crystalline terranes. Geology 12, 87-90.

St.-Onge, M. R., 1984. Geothermometry and geobarometry in pelitic rocks of north-central Wopmay Orogen (early Proterozoic), Northwest Territories, Canada. Geol. Soc. Am. Bull. 95, 196-208.

- 1987. Zoned poikiloblastic garnets: $P-T$ paths and syn-metamorphic uplift through $30 \mathrm{~km}$ of structural depth, Wopmay Orogen, Canada. J. Petrology 28, 1-21.

King, J. E., 1987. Thermo-tectonic evolution of a metamorphic internal zone documented by axial projections and petrological $P-T$ paths, Wopmay orogen, northwest Canada. Geology 15, 155-8.

Thompson, A. B, \& England, P. C., 1984. Pressure-temperature-time paths of regional metamorphism. II. Their inference and interpretation using mineral assemblages in metamorphic rocks. $J$. Petrology 25, 929-55.

Tracy, R. J., 1982. Compositional zoning and inclusions in metamorphic minerals. In: Ferry, J. M. (ed.) Characterization of Metamorphism Through Mineral Equilibria. Miner. Soc. Am. Rev. Miner. 10, 355-97.

- Robinson, P., \& Thompson, A. B., 1976. Gamet composition and zoning in the determination of temperature and pressure of metamorphism, central Massachusetts. Am. Miner. 61, 762-75.

Tuccillo, M. E., 1990. Pressure-temperature-time history of the Britt domain, Ontario Grenville Province. Unpublished Master's Thesis, University of Michigan. 
- Essene, E. J., \& van der Pluijm, B. A., 1990. Growth and retrograde zoning in garnets from high-grade metapelites: implications for pressure-temperature paths. Geology 18, 839-42.

van Breemen, A., Davidson, A., Loveridge, W. D., \& Sullivan, R. W., 1986. U-Pb zircon geochronology of Grenville tectonites, granulites and igneous precursors, Parry Sound, Ontario. In: Moore, J. M, Davidson, A., \& Baer, A. J. (eds.) The Grenville Province. Geol. Assac. Can. Spec. Paper 31, 191-207.

Woodsworth, G. J, 1977. Homogenization of zoned garnets from pelitic schists. Can. Miner. 15, 230-42.

Wynne-Edwards, H. R., 1972. The Grenville Province. In: Price, R. A., \& Douglas, R. J. W. (eds.) Variations in Tectonic Styles in Canada. Geol. Assoc. Can. Spec. Paper 11, 263-334.

Yardley, B. W. D, 1977. An empirical study of diffusion in garnet. Am. Miner. 62, 793-800.

Young, E. D., Anderson, J. L., Clarke, H. S, \& Thomas, W. M., 1989. Petrology of biotite-cordierite-gamet gneiss of the McCullough Range, Nevada. 1. Evidence for Proterozoic low-pressure fluid-absent granulite metamorphism in the southern Cordillera. J. Petrology 30, 39-60.

A PPENDIX A

Sample locations

\begin{tabular}{lccc}
\hline Sample ID & Sample no. & Northing & Easting \\
\hline MET88-41 & 1 & 50164 & 5583 \\
MET88-52 & 2 & 50417 & 5566 \\
NC25 & 3 & 50250 & 5510 \\
PS88-1 & 4 & 50225 & 5738 \\
PSO82a-4a & 5 & 50128 & 5725 \\
Sh88-1 & 6 & 50427 & 5574 \\
Sh88-2a & 7 & 50440 & 5560 \\
Sh88-7b & 8 & 50410 & 5502 \\
Sh88-9a & 9 & 50464 & 5562 \\
Sh88-12 & 10 & 50451 & 5542 \\
Sh88-42 & 11 & 50416 & 5483 \\
$88 D M G-D 047 a$ & 12 & 50370 & 5545 \\
91-16 & 13 & 50290 & 5710 \\
91-18 & 14 & 50225 & 5738 \\
Sh88-58 & 15 & 50410 & 5502 \\
91-19 & 16 & 50366 & 5816 \\
\hline
\end{tabular}

Sample locations are given in terms of a UTM grid. All locations are in Zone 17 and are given in values of $100 \mathrm{~m}$. 